\title{
C-H Polyaddition of Dimethoxyarenes to Unconjugated Dienes by Rare Earth Catalysts
}

\author{
Xiaochao Shi, Masayoshi Nishiura, and Zhaomin Hou* \\ Organometallic Chemistry Laboratory and Center for Sustainable Resource Science, RIKEN, 2-1 \\ Hirosawa, Wako, Saitama 351-0198, Japan \\ E-mail: houz@riken.jp
}




\section{Experimental Section}

Table S1. Copolymerization of 1,4-dimethoxybenzene with 2,5-norbornadiene by various rare earth catalysts

Scheme S1. Polyaddition of 1,4-dibutoxybenzene to 4,4'-divinylbiphenyl

Scheme S2. Polyaddition of 1,4-dimethoxybenzene to 1,9-decadiene

Figure S1. ${ }^{1} \mathrm{H}$ NMR spectrum $\left(500 \mathrm{MHz}, \mathrm{C}_{2} \mathrm{D}_{2} \mathrm{Cl}_{4}, 120{ }^{\circ} \mathrm{C}\right)$ of copoly(1,4-dimethoxybenzene/ norbornadiene) (Table 1, Entry 6)

Figure S2. ${ }^{13} \mathrm{C}$ NMR spectrum $\left(125 \mathrm{MHz}, \mathrm{C}_{2} \mathrm{D}_{2} \mathrm{Cl}_{4}, 120{ }^{\circ} \mathrm{C}\right)$ of copoly(1,4-dimethoxybenzene/ norbornadiene) (Table 1, Entry 6)

Figure S3. DEPT $135-{ }^{13} \mathrm{C}$ spectrum $\left(125 \mathrm{MHz}, \mathrm{C}_{2} \mathrm{D}_{2} \mathrm{Cl}_{4}, 120{ }^{\circ} \mathrm{C}\right)$ of copoly(1,4-dimethoxybenzene/ norbornadiene) (Table 1, Entry 6)

Figure S4. HSQC spectrum $\left(\mathrm{C}_{2} \mathrm{D}_{2} \mathrm{Cl}_{4}, 120^{\circ} \mathrm{C}\right)$ of copoly(1,4-dimethoxybenzene/norbornadiene) (Table 1, Entry 6)

Figure S5. HMBC spectrum $\left(\mathrm{C}_{2} \mathrm{D}_{2} \mathrm{Cl}_{4}, 120^{\circ} \mathrm{C}\right)$ of copoly(1,4-dimethoxybenzene/norbornadiene) (Table 1, Entry 6)

Figure S6. ${ }^{1} \mathrm{H}$ NMR spectrum of copoly(1,4-dimethoxybenzene/norbornadiene) in different polymerization time (Table 1, Entries 1-6)

Figure S7. ${ }^{1} \mathrm{H}$ NMR spectrum $\left(500 \mathrm{MHz}, \mathrm{C}_{2} \mathrm{D}_{2} \mathrm{Cl}_{4}, 120{ }^{\circ} \mathrm{C}\right)$ of copoly(1,4-dimethoxybenzene/1,4divinylbenzene) (Table 2, Entry 3)

Figure S8. ${ }^{13} \mathrm{C}$ NMR spectrum $\left(125 \mathrm{MHz}, \mathrm{C}_{2} \mathrm{D}_{2} \mathrm{Cl}_{4}, 120{ }^{\circ} \mathrm{C}\right)$ of copoly(1,4-dimethoxybenzene/1,4divinylbenzene) (Table 2, Entry 3 )

Figure S9. DEPT135- ${ }^{13} \mathrm{C}$ spectrum $\left(\begin{array}{llllll}125 & \mathrm{MHz}, & \mathrm{C}_{2} \mathrm{D}_{2} \mathrm{Cl}_{4}, & 120 & { }^{\circ} \mathrm{C}\end{array}\right)$ of copoly $(1,4-$ dimethoxybenzene/1,4-divinylbenzene) (Table 2, Entry 3)

Figure S10. HSQC spectrum $\left(\mathrm{C}_{2} \mathrm{D}_{2} \mathrm{Cl}_{4}, \quad 120{ }^{\circ} \mathrm{C}\right) \quad$ of copoly(1,4-dimethoxybenzene/1,4divinylbenzene) (Table 2, Entry 3)

Figure S11. HMBC spectrum $\left(\mathrm{C}_{2} \mathrm{D}_{2} \mathrm{Cl}_{4}, \quad 120{ }^{\circ} \mathrm{C}\right)$ of copoly(1,4-dimethoxybenzene/1,4divinylbenzene) (Table 2, Entry 3)

Figure S12. ${ }^{1} \mathrm{H}$ NMR spectrum $\left(500 \mathrm{MHz}, \mathrm{C}_{2} \mathrm{D}_{2} \mathrm{Cl}_{4}, 120^{\circ} \mathrm{C}\right)$ of copoly(1,4-dimethoxybenzene/4,4'divinylbiphenyl)

Figure S13. ${ }^{13} \mathrm{C}$ NMR spectrum $\left(125 \mathrm{MHz}, \mathrm{C}_{2} \mathrm{D}_{2} \mathrm{Cl}_{4}, 120{ }^{\circ} \mathrm{C}\right)$ of copoly(1,4-dimethoxybenzene/4,4'divinylbiphenyl)

Figure S14. DEPT $135-{ }_{-}^{13} \mathrm{C}$ spectrum $\left(125 \mathrm{MHz}, \mathrm{C}_{2} \mathrm{D}_{2} \mathrm{Cl}_{4}, 120{ }^{\circ} \mathrm{C}\right)$ of copoly(1,4-dimethoxybenzene/4,4'divinylbiphenyl)

Figure S15. HSQC spectrum $\left(\mathrm{C}_{2} \mathrm{D}_{2} \mathrm{Cl}_{4}, 120^{\circ} \mathrm{C}\right)$ of copoly(1,4-dimethoxybenzene/4,4'-divinylbiphenyl)

Figure S16. $\mathrm{HMBC}$ spectrum $\left(\mathrm{C}_{2} \mathrm{D}_{2} \mathrm{Cl}_{4}, 120^{\circ} \mathrm{C}\right)$ of copoly(1,4-dimethoxybenzene/4,4'-divinylbiphenyl)

Figure S17. ${ }^{1} \mathrm{H}$ NMR spectrum $\left(500 \mathrm{MHz}, \mathrm{C}_{2} \mathrm{D}_{2} \mathrm{Cl}_{4}, 120{ }^{\circ} \mathrm{C}\right)$ of copoly $\left(4,4^{\prime}\right.$-dimethoxybiphenyl/ norbornadiene)

Figure S18. ${ }^{13} \mathrm{C}$ NMR spectrum $\left(125 \mathrm{MHz}, \mathrm{C}_{2} \mathrm{D}_{2} \mathrm{Cl}_{4}, 120{ }^{\circ} \mathrm{C}\right)$ of copoly $\left(4,4^{\prime}\right.$-dimethoxybiphenyl/ norbornadiene) 
Figure S19. DEPT135- ${ }^{13} \mathrm{C}$ spectrum $\left(125 \mathrm{MHz}, \mathrm{C}_{2} \mathrm{D}_{2} \mathrm{Cl}_{4}, 120{ }^{\circ} \mathrm{C}\right)$ of copoly $\left(4,4^{\prime}\right.$-dimethoxybiphenyl/ norbornadiene)

Figure S20. HSQC spectrum $\left(\mathrm{C}_{2} \mathrm{D}_{2} \mathrm{Cl}_{4}, 120^{\circ} \mathrm{C}\right)$ of copoly $\left(4,4^{\prime}\right.$-dimethoxybiphenyl/norbornadiene $)$

Figure S21. $\mathrm{HMBC}$ spectrum $\left(\mathrm{C}_{2} \mathrm{D}_{2} \mathrm{Cl}_{4}, 120^{\circ} \mathrm{C}\right)$ of copoly(4,4'-dimethoxybiphenyl/norbornadiene)

Figure S22. ${ }^{1} \mathrm{H}$ NMR spectrum $\left(500 \mathrm{MHz}, \mathrm{C}_{2} \mathrm{D}_{2} \mathrm{Cl}_{4}, 120{ }^{\circ} \mathrm{C}\right)$ of copoly $\left(4,4^{\prime}\right.$-dimethoxybiphenyl/1,4divinylbenzene)

Figure S23. ${ }^{13} \mathrm{C}$ NMR spectrum $\left(125 \mathrm{MHz}, \mathrm{C}_{2} \mathrm{D}_{2} \mathrm{Cl}_{4}, 120{ }^{\circ} \mathrm{C}\right)$ of copoly $\left(4,4^{\prime}\right.$-dimethoxybiphenyl/1,4divinylbenzene)

Figure S24. DEPT $135-{ }^{13} \mathrm{C}$ spectrum $\left(125 \mathrm{MHz}, \mathrm{C}_{2} \mathrm{D}_{2} \mathrm{Cl}_{4}, 120{ }^{\circ} \mathrm{C}\right)$ of copoly $\left(4,4^{\prime}\right.$-dimethoxybiphenyl/1,4divinylbenzene)

Figure S25. HSQC spectrum $\left(\mathrm{C}_{2} \mathrm{D}_{2} \mathrm{Cl}_{4}, 120^{\circ} \mathrm{C}\right)$ of copoly (4,4'-dimethoxybiphenyl/1,4-divinylbenzene)

Figure S26. $\mathrm{HMBC}$ spectrum $\left(\mathrm{C}_{2} \mathrm{D}_{2} \mathrm{Cl}_{4}, 120^{\circ} \mathrm{C}\right)$ of copoly(4,4'-dimethoxybiphenyl/1,4-divinylbenzene)

Figure S27. ${ }^{1} \mathrm{H}$ NMR spectrum $\left(500 \mathrm{MHz}, \mathrm{C}_{2} \mathrm{D}_{2} \mathrm{Cl}_{4}, 120{ }^{\circ} \mathrm{C}\right)$ of copoly(1,4-dibutoxybenzene/4,4'divinylbiphenyl)

Figure S28. ${ }^{13} \mathrm{C}$ NMR spectrum $\left(125 \mathrm{MHz}, \mathrm{C}_{2} \mathrm{D}_{2} \mathrm{Cl}_{4}, 120{ }^{\circ} \mathrm{C}\right)$ of copoly(1,4-dibutoxybenzene/4,4'divinylbiphenyl)

Figure S29. DEPT135- ${ }^{13} \mathrm{C}$ spectrum $\left(125 \mathrm{MHz}, \mathrm{C}_{2} \mathrm{D}_{2} \mathrm{Cl}_{4}, 120{ }^{\circ} \mathrm{C}\right)$ of copoly(1,4-dibutoxybenzene/4,4'divinylbiphenyl)

Figure S30. HSQC spectrum $\left(\mathrm{C}_{2} \mathrm{D}_{2} \mathrm{Cl}_{4}, 120^{\circ} \mathrm{C}\right)$ of copoly(1,4-dibutoxybenzene/4,4'-divinylbiphenyl)

Figure S31. HMBC spectrum $\left(\mathrm{C}_{2} \mathrm{D}_{2} \mathrm{Cl}_{4}, 120^{\circ} \mathrm{C}\right)$ of copoly(1,4-dibutoxybenzene/4,4'-divinylbiphenyl)

Figure S32. ${ }^{1} \mathrm{H}$ NMR spectrum $\left(500 \mathrm{MHz}, \mathrm{C}_{2} \mathrm{D}_{2} \mathrm{Cl}_{4}, 26.8{ }^{\circ} \mathrm{C}\right)$ of copoly $(1,4-$ dimethoxybenzene/1,9decadiene)

Figure S33. ${ }^{13} \mathrm{C}$ NMR spectrum $\left(125 \mathrm{MHz}, \mathrm{C}_{2} \mathrm{D}_{2} \mathrm{Cl}_{4}, 26.8{ }^{\circ} \mathrm{C}\right)$ of copoly(1,4-dimethoxybenzene/1,9decadiene)

Figure S34. DEPT $135-{ }^{13} \mathrm{C}$ spectrum $\left(125 \mathrm{MHz}, \quad \mathrm{C}_{2} \mathrm{D}_{2} \mathrm{Cl}_{4}, \quad 26.8 \quad{ }^{\circ} \mathrm{C}\right) \quad$ of $\operatorname{copoly}(1,4-$ dimethoxybenzene/1,9-decadiene)

Figure S35. HSQC spectrum $\left(\mathrm{C}_{2} \mathrm{D}_{2} \mathrm{Cl}_{4}, 26.8^{\circ} \mathrm{C}\right)$ of copoly(1,4-dimethoxybenzene/1,9-decadiene)

Figure S36. HMBC spectrum $\left(\mathrm{C}_{2} \mathrm{D}_{2} \mathrm{Cl}_{4}, 26.8^{\circ} \mathrm{C}\right)$ of copoly (1,4-dimethoxybenzene/1,9-decadiene)

Figure S37. GPC curve of copoly(1,4-dimethoxybenzene/norbornadiene) $\left(M_{\mathrm{n}}=9800, M_{\mathrm{w}} / M_{\mathrm{n}}=2.00\right)$ in Table 1, Entry 6

Figure S38 GPC curve of of copoly(1,4-dimethoxybenzene/1,4-divinylbenzene) $\left(M_{\mathrm{n}}=3180, M_{\mathrm{w}} / M_{\mathrm{n}}=\right.$ 2.38) in Table 2, Entry 3

Figure S39. GPC curve of copoly(1,4-dimethoxybenzene/4,4'-divinylbiphenyl) ( $M_{\mathrm{n}}=1900, M_{\mathrm{w}} / M_{\mathrm{n}}$ $=1.94)$

Figure S40. GPC curve of copoly(4,4'-dimethoxybiphenyl/norbornadiene) $\left(M_{\mathrm{n}}=1330, M_{\mathrm{w}} / M_{\mathrm{n}}=1.91\right)$

Figure S41. GPC curve of copoly(4,4'-dimethoxybiphenyl/1,4-divinylbenzene) $\left(M_{\mathrm{n}}=4550, M_{\mathrm{w}} / M_{\mathrm{n}}=\right.$ 2.52)

Figure S42. GPC curve of copoly(1,4-dimethoxybenzene/1,9-decadiene) $\left(M_{\mathrm{n}}=2160, M_{\mathrm{w}} / M_{\mathrm{n}}=1.23\right)$ 
Figure S43. DSC curve of copoly(1,4-dimethoxybenzene/norbornadiene) (Table 1, Entry 6)

Figure S44. DSC curve of copoly(1,4-dimethoxybenzene/1,4-divinylbenzene) (Table 2, Entry 3)

Figure S45. DSC curve of copoly(1,4-dimethoxybenzene/4,4'-divinylbiphenyl)

Figure S46. DSC curve of copoly(4,4'-dimethoxybiphenyl/norbornadiene)

Figure S47. DSC curve of copoly(4,4'-dimethoxybiphenyl/1,4-divinylbenzene)

Figure S48. DSC curve of copoly(1,4-dimethoxybenzene/1,9-decadiene) 


\section{Experimental Section}

General Procedure and Materials All manipulations of air and moisture-sensitive compounds were performed under a dry nitrogen atmosphere by use of standard Schlenk techniques or a nitrogen-filled Mbraun glovebox. Nitrogen was purified by being passed through a Dryclean column DC-A4 (4 $\AA$ molecular sieves, Nikka Seiko Co.) and a Gasclean GC-XR column (Nikka Seiko Co.). Solvents were purified by an Mbraun SPS-800 Solvent Purification System and dried over fresh Na chips in the glovebox. Norbornadiene and 1,9-decadiene were purchased from Kanto Chemical Co. Int and Tokyo Chemical Ind. Co. Ltd, dried over $\mathrm{CaH}_{2}$, and vacuum-transferred prior to polymerization experiments. $\left[\mathrm{Ph}_{3} \mathrm{C}\right]\left[\mathrm{B}\left(\mathrm{C}_{6} \mathrm{~F}_{5}\right)_{4}\right]$ was purchased from Strem Chemical Corporation and used without purification. $\mathrm{Cp}^{\prime} \mathrm{Ln}\left(\mathrm{CH}_{2} \mathrm{C}_{6} \mathrm{H}_{4} \mathrm{NMe}_{2}-\mathrm{O}\right)_{2}\left(\mathrm{Cp}^{\prime}=\mathrm{C}_{5} \mathrm{Me}_{5}, \mathrm{C}_{5} \mathrm{Me}_{4} \mathrm{SiMe}_{3} ; \mathrm{Ln}=\mathrm{Sc}, \mathrm{Y}, \mathrm{Gd}, \mathrm{Sm}, \mathrm{Lu}\right),{ }^{1-2}$ 1,4-divinylbenzene and $4,4^{\prime}$-divinylbiphenyl ${ }^{3}$ were synthesized according to literatures. The deuterated solvents benzene- $\mathrm{d}_{6}$ (99.6 atom \% D), THF- $\mathrm{d}_{8}\left(99.6\right.$ atom \% D), $\mathrm{CDCl}_{3}\left(99.8\right.$ atom \% D), and 1,1,2,2-tetrachloroethane- $\mathrm{d}_{2}$ (99.6 atom \% D) were obtained from Kanto Chemical Co. Inc. and Cambridge Isotope. The NMR data of the polymers were obtained on a Bruker AVANCE III HD 500 NMR (FT, $500 \mathrm{MHz}$ for ${ }^{1} \mathrm{H} ; 125 \mathrm{MHz}$ for ${ }^{13} \mathrm{C}$ ) spectrometer at $120{ }^{\circ} \mathrm{C}$ or $26.8^{\circ} \mathrm{C}$ with $1,1,2,2-\mathrm{C}_{2} \mathrm{D}_{2} \mathrm{Cl}_{4}$ as a solvent. The molecular weights and the molecular weight distributions of all the copolymers were determined at $145^{\circ} \mathrm{C}$ by high temperature gel permeation chromatography (HT-GPC) on a HLC-8121GPC/HT apparatus (Tosoh Corporation). 1,2Dichlorobenzene (DCB) was employed as an eluent at a flow rate of $1.0 \mathrm{~mL} / \mathrm{min}$. The molecular weights and the molecular weight distributions of copoly(1,4-dimethoxybenzene/1,9-decadiene) was determined at $40{ }^{\circ} \mathrm{C}$ by gel permeation chromatography (GPC) on a HLC-8320 GPC apparatus (Tosoh Corporation), THF was employed as an eluent at a flow rate of $0.35 \mathrm{~mL} / \mathrm{min}$. The calibration was made by use of polystyrene standard (Tosoh Corporation). The DSC measurements were performed on a DSC 6220 (S II Corporation) at a rate of $10{ }^{\circ} \mathrm{C} / \mathrm{min}$. Any thermal history difference in the polymers was eliminated by first heating the specimen to $300{ }^{\circ} \mathrm{C}$ (or $250{ }^{\circ} \mathrm{C}$ ), cooling at $10{ }^{\circ} \mathrm{C} / \mathrm{min}$ to $-10{ }^{\circ} \mathrm{C}$ (or $-100{ }^{\circ} \mathrm{C}$ ), and then recording the second DSC scan. 


\section{A Typical Procedure for the Polyaddition of dimethoxybenzene to norbornadiene (Table S1, Entry}

1). In a glovebox, a toluene solution $(1.0 \mathrm{~mL})$ of $\left[\mathrm{Ph}_{3} \mathrm{C}\right]\left[\mathrm{B}\left(\mathrm{C}_{6} \mathrm{~F}_{5}\right)_{4}\right](23.1 \mathrm{mg}, 0.025 \mathrm{mmol})$ was slowly

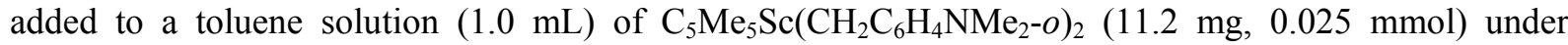
magnetic stirring in a $20 \mathrm{~mL}$ Schlenk tube. After the mixture was stirred for $5 \mathrm{~min}$, dimethoxybenzene (138 mg, $1.0 \mathrm{mmol})$ and norbornadiene $(92 \mathrm{mg}, 1.0 \mathrm{mmol})$ were added. The Schlenk tube was sealed and taken out of the glovebox and then heated at $70{ }^{\circ} \mathrm{C}$ for 24 hours. The polymerization was quenched by addition of methanol (50 mL, containing $5 \%$ butylhydroxytoluene (BHT) as a stabilizing agent). Then the mixture was poured into methanol $(200 \mathrm{~mL})$ to precipitate the polymer product. The precipitated polymer was dried under vacuum at $60{ }^{\circ} \mathrm{C}$ to a constant weight ( $230 \mathrm{mg}, 100 \%$ yield).

The experimental results of the copolymerization of 1,4-dimethoxybenzene with 2,5-norbornadiene by various rare earth catalysts are summarized in Table S1.

Table S1. Copolymerization of 1,4-dimethoxybenzene with 2,5-norbornadiene by various rare earth catalysts $^{a}$

\begin{tabular}{ccccccc}
\hline Entry & $\operatorname{Ln}^{a}$ & Yield $^{b}(\%)$ & $2,5 / 2,6^{c}$ & $M_{\mathrm{n}}\left(\times 10^{3}\right)^{d}$ & $M_{\mathrm{w}} / M_{\mathrm{n}}{ }^{d}$ & $\mathrm{~T}_{\mathrm{g}}{ }^{e}\left({ }^{0} \mathrm{C}\right)$ \\
\hline 1 & $\mathbf{1 - S c}$ & 100 & 1.21 & 9.80 & 2.00 & 199 \\
2 & $\mathbf{1}-\mathbf{Y}$ & 0 & - & - & - & - \\
3 & $\mathbf{1 - S m}$ & 0 & - & - & - & - \\
4 & $\mathbf{1 - G d}$ & 0 & - & - & - & - \\
5 & $\mathbf{1 - L u}$ & 19 & n.d. & 0.62 & 1.79 & n.d. \\
6 & $\mathbf{2 - S c}$ & 64 & 1.20 & 1.63 & 1.59 & 127 \\
7 & $\mathbf{2 - Y}$ & 0 & - & - & - & - \\
\hline
\end{tabular}

${ }^{a}$ Reaction conditions: [M] $(0.025 \mathrm{mmol}),\left[\mathrm{Ph}_{3} \mathrm{C}\right]\left[\mathrm{B}\left(\mathrm{C}_{6} \mathrm{~F}_{5}\right)_{4}\right](0.025 \mathrm{mmol}), 1,4$-dimethoxybenzene (1 mmol): norbornadiene $(1 \mathrm{mmol})$, toluene $(2 \mathrm{~mL}), 70{ }^{\circ} \mathrm{C}, 24 \mathrm{~h} ;{ }^{b}$ Isolated yield; ${ }^{c}$ Calculated from the ${ }^{13} \mathrm{C}$ NMR spectrum; ${ }^{d}$ Determined by GPC against polystyrene standards at $145{ }^{\circ} \mathrm{C}$ in $o$-dichlorobenzene; ${ }^{e}$ Measured by DSC.

(1) Li, X.; Nishiura, M.; Mori, K.; Mashiko, T.; Hou, Z. Chem. Commun. 2007, 4137.

(2) Nishiura, M.; Baldamus, J.; Shima, T.; Mori, K.; Hou, Z. Chem. Eur. J. 2011, 17, 5033.

(3) Ludwiczak, M.; Majchrzak, M.; Marciniec, B.; Kubicki, M. J. Organomet. Chem. 2011, 696, 1456. 
The copolymerization of 1,4-dibutoxybenzene (DBB) with 4,4'-divinylbiphenyl (DVBP) (Scheme S1) and 1,4-dimethoxybenzene (DMB) with 1,9-decadiene (Scheme S2) were carried out in a similar fashion.

\section{Scheme S1. C-H Polyaddition of 1,4-dibutoxybenzene to 4,4'-divinylbiphenyl}

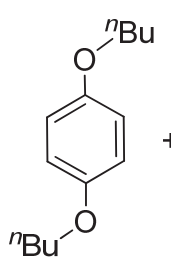

(DBB)

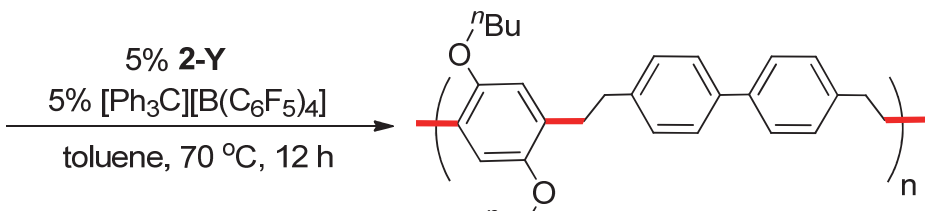

"Bu' yield $67 \%, M_{\mathrm{n}}=2570, M_{\mathrm{w}} / M_{\mathrm{n}}=2.61$

Scheme S2. C-H Polyaddition of 1,4-dimethoxybenzene to 1,9-decadiene

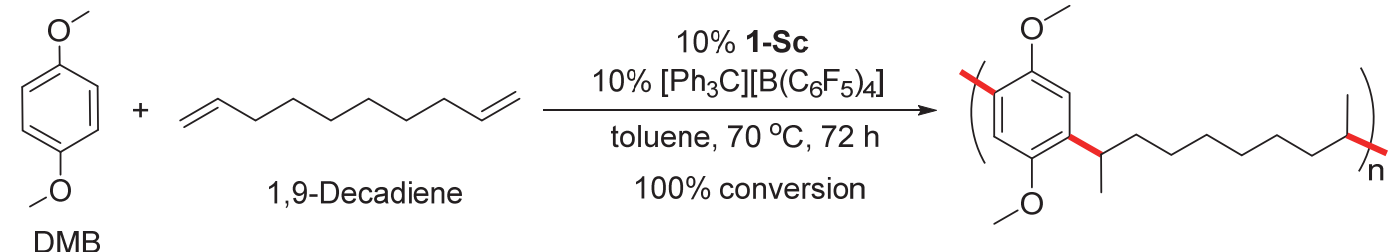

$26 \%$ (MeOH-insoluble), $M_{\mathrm{n}}=2160, M_{\mathrm{w}} / M_{\mathrm{n}}=1.23, T_{\mathrm{g}}=-16^{\circ} \mathrm{C}$ $74 \%$ (MeOH-soluble), $M_{\mathrm{n}}=470, M_{\mathrm{w}} / M_{\mathrm{n}}=2.27$ 


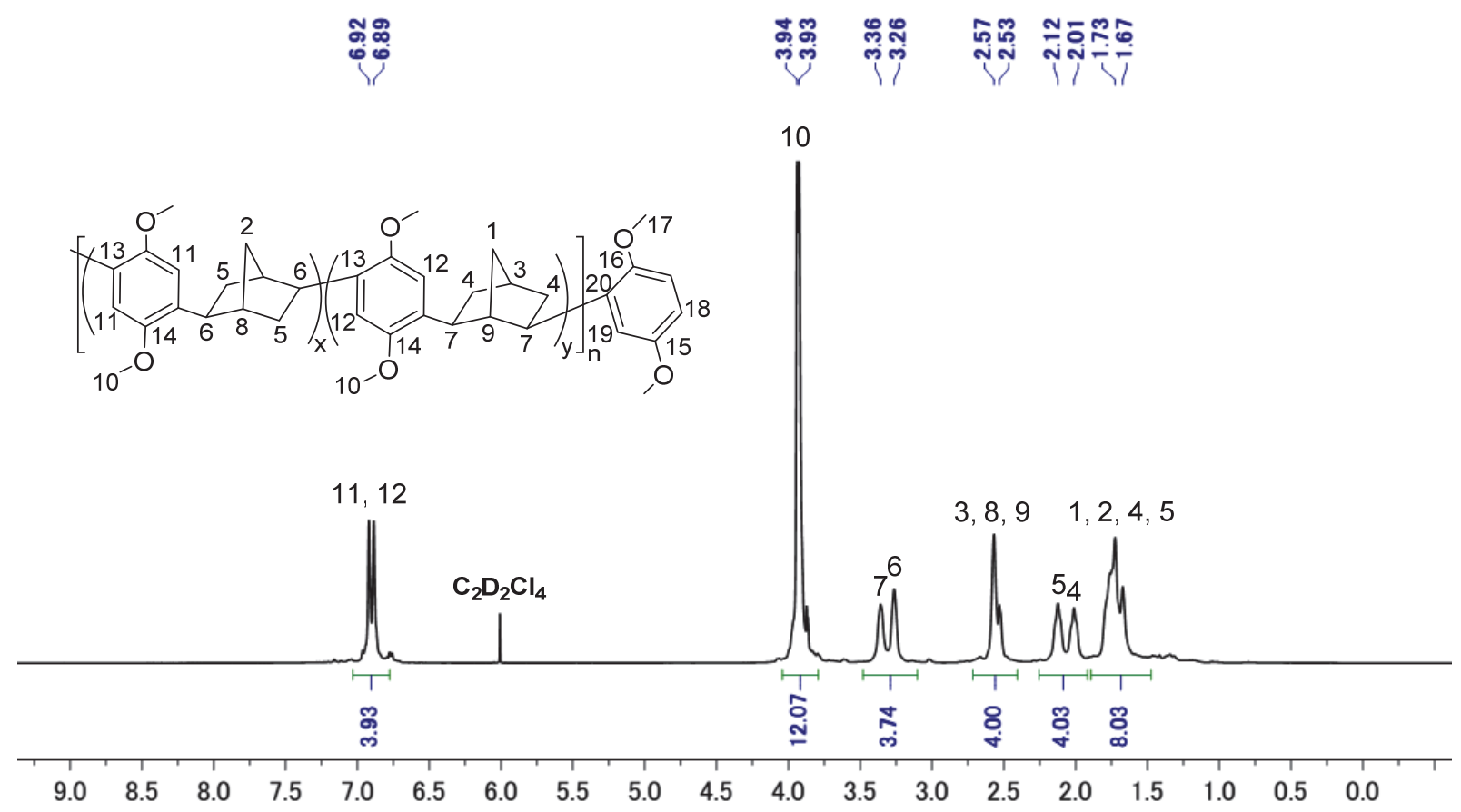

Figure S1. ${ }^{1} \mathrm{H}$ NMR spectrum $\left(500 \mathrm{MHz}, \mathrm{C}_{2} \mathrm{D}_{2} \mathrm{Cl}_{4}, 120{ }^{\circ} \mathrm{C}\right)$ of copoly(1,4-dimethoxybenzene/ norbornadiene) (Table 1, Entry 6)

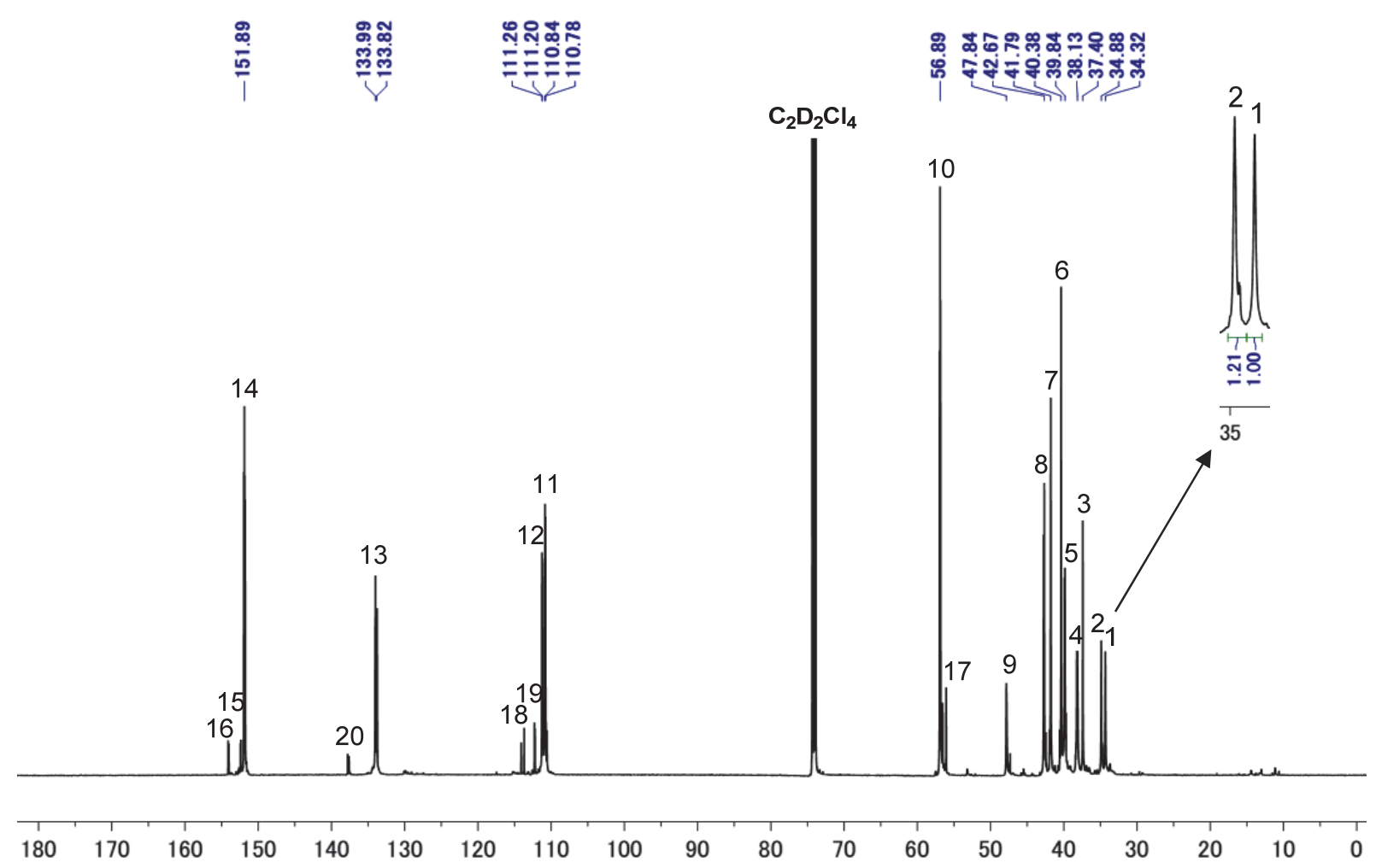

Figure S2. ${ }^{13} \mathrm{C}$ NMR spectrum $\left(125 \mathrm{MHz}, \mathrm{C}_{2} \mathrm{D}_{2} \mathrm{Cl}_{4}, 120{ }^{\circ} \mathrm{C}\right)$ of copoly(1,4-dimethoxybenzene/ norbornadiene) (Table 1, Entry 6) 


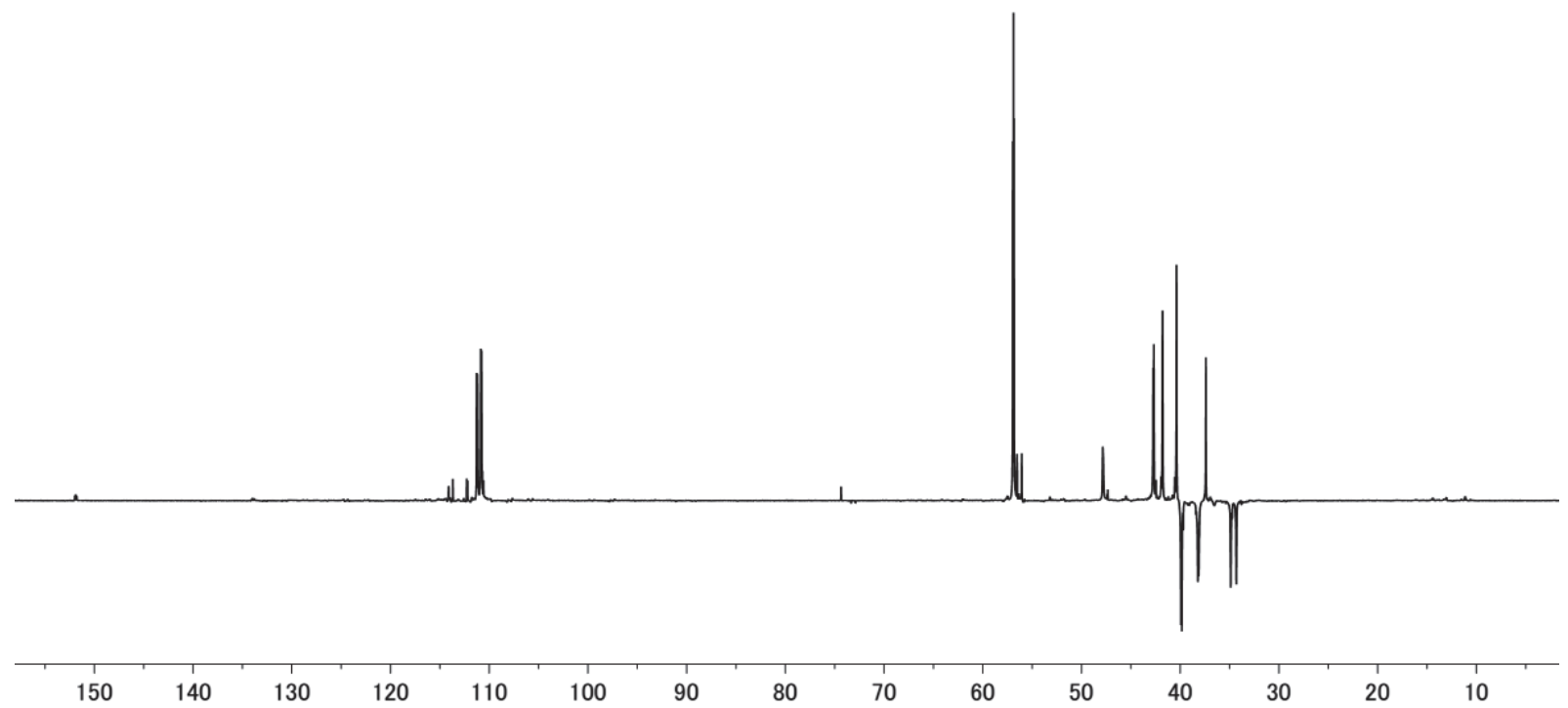

Figure S3. DEPT $135-{ }^{13} \mathrm{C}$ spectrum $\left(125 \mathrm{MHz}, \mathrm{C}_{2} \mathrm{D}_{2} \mathrm{Cl}_{4}, 120{ }^{\circ} \mathrm{C}\right)$ of copoly(1,4-dimethoxybenzene/ norbornadiene) (Table 1, Entry 6)

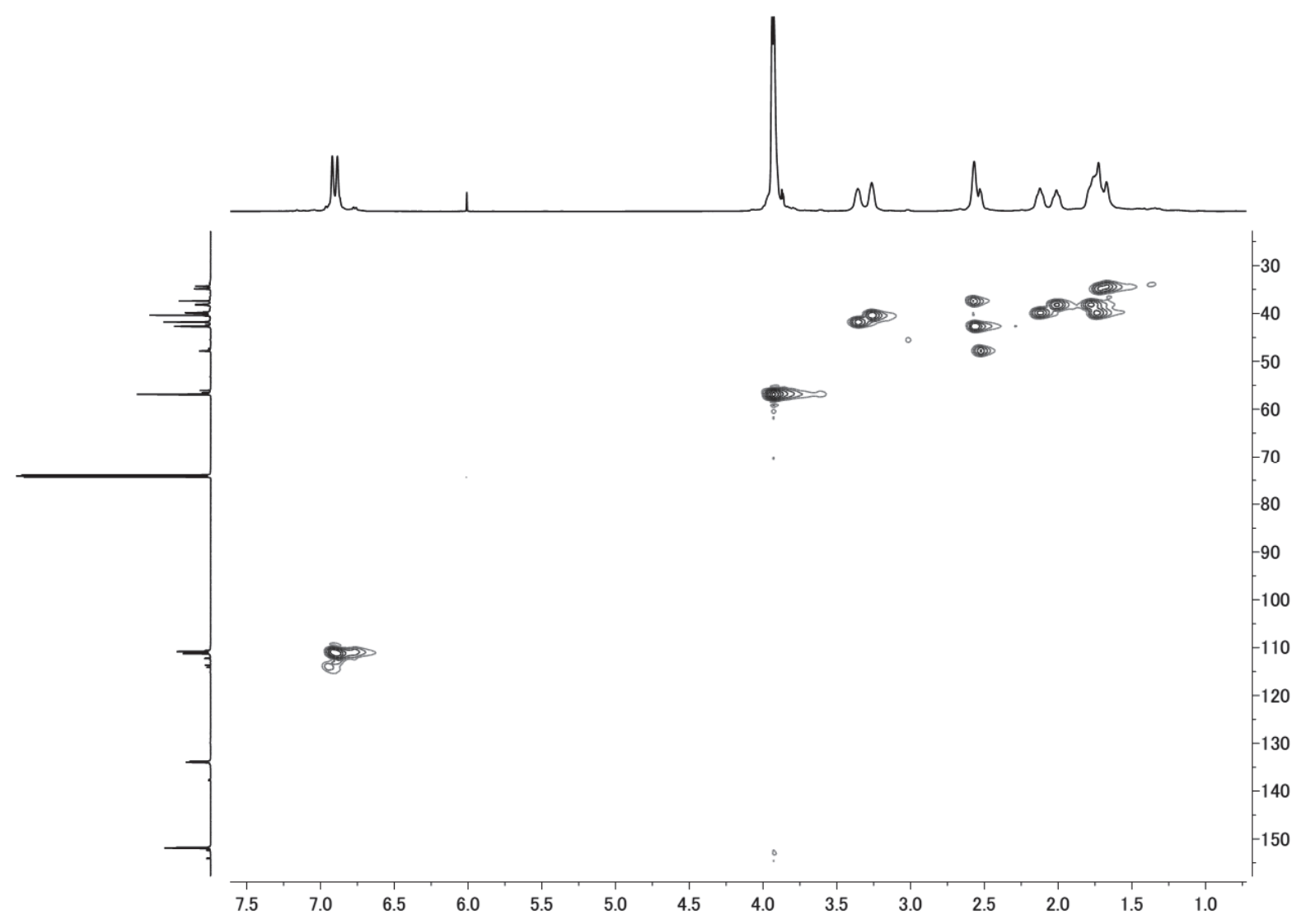

Figure S4. HSQC spectrum $\left(\mathrm{C}_{2} \mathrm{D}_{2} \mathrm{Cl}_{4}, 120^{\circ} \mathrm{C}\right)$ of copoly(1,4-dimethoxybenzene/norbornadiene) (Table 1, Entry 6) 


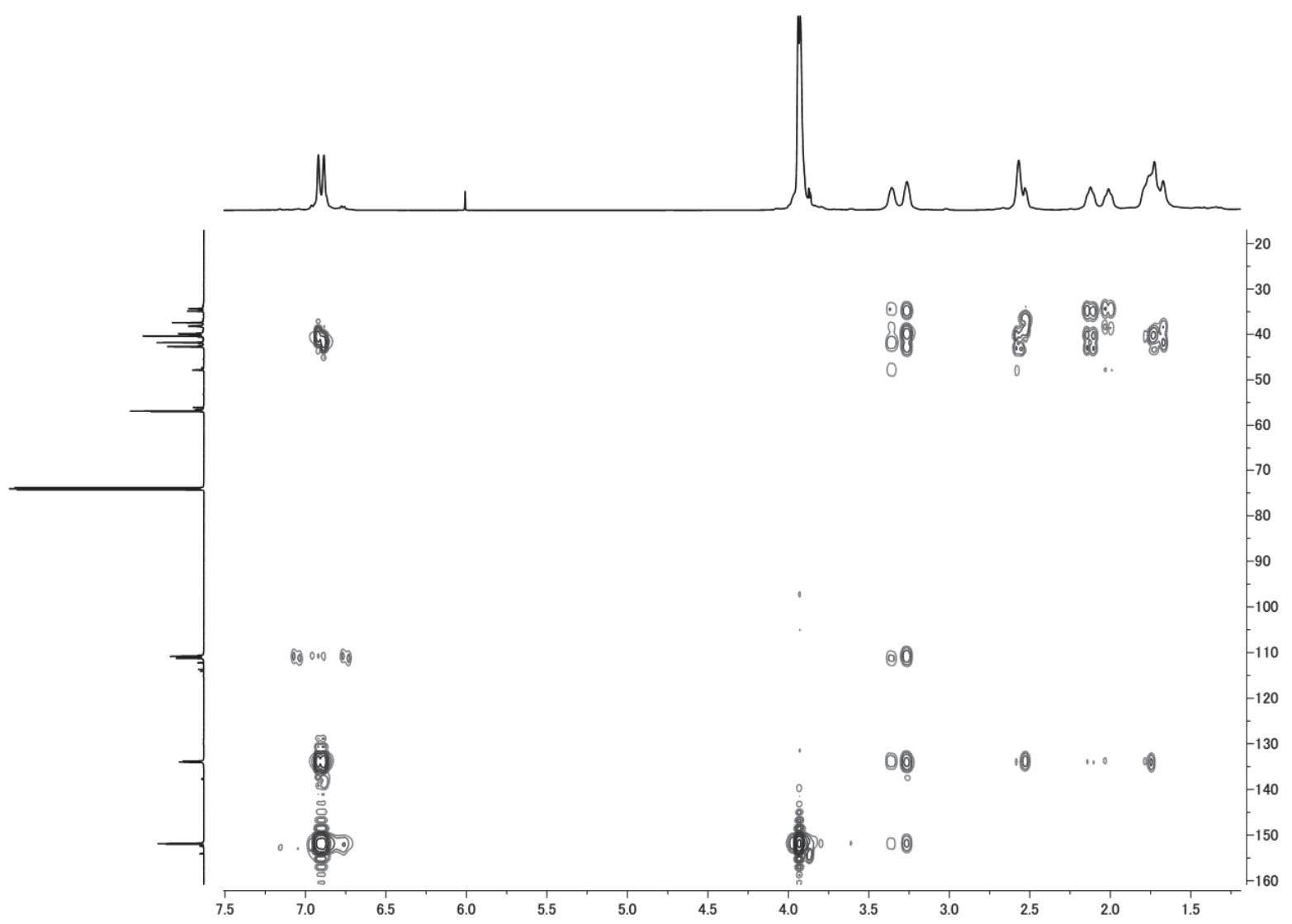

Figure S5. HMBC spectrum $\left(\mathrm{C}_{2} \mathrm{D}_{2} \mathrm{Cl}_{4}, 120^{\circ} \mathrm{C}\right)$ of copoly(1,4-dimethoxybenzene/norbornadiene) (Table 1, Entry 6) 


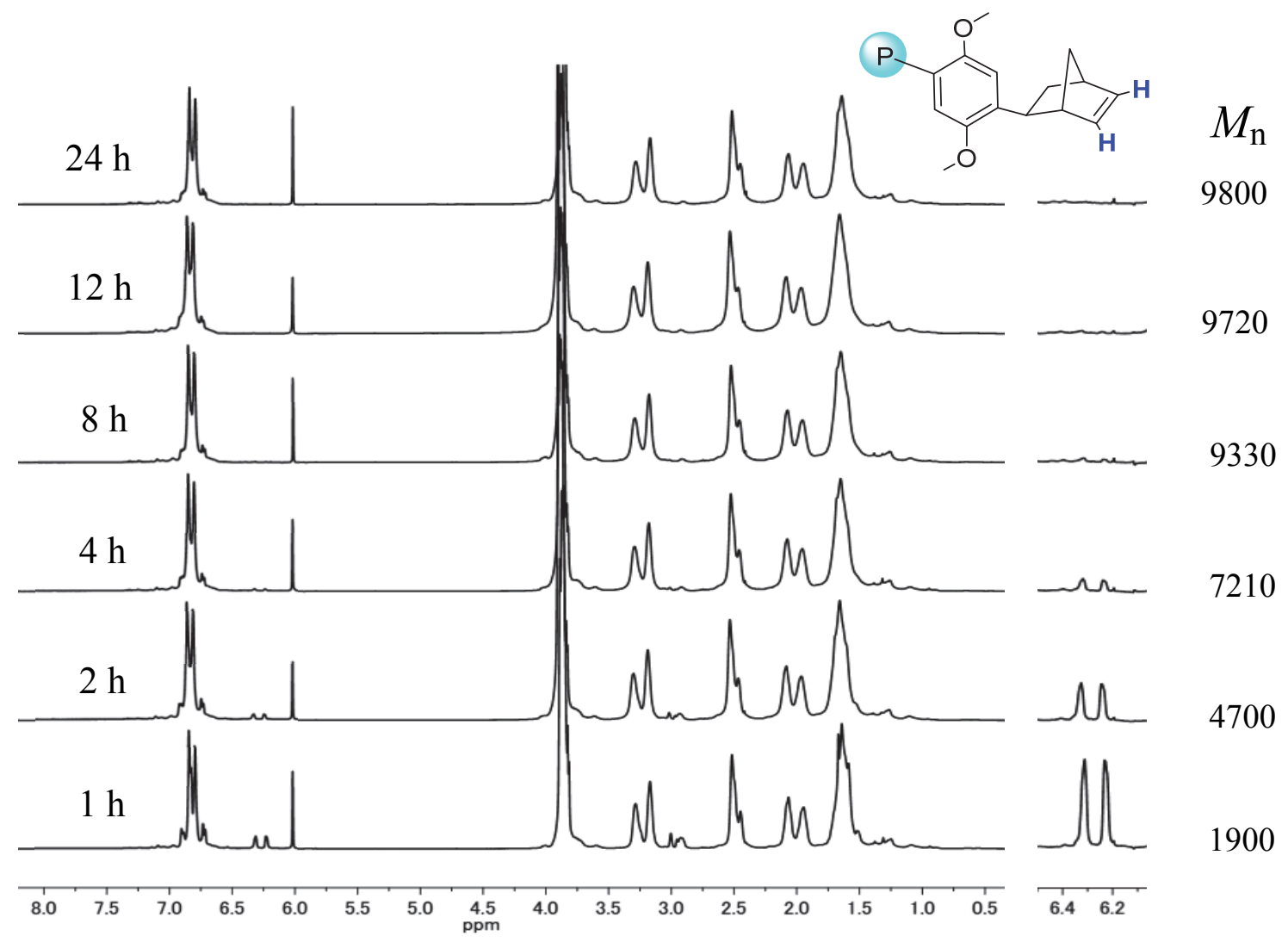

Figure S6. ${ }^{1} \mathrm{H}$ NMR spectrum of copoly(1,4-dimethoxybenzene/norbornadiene) in different polymerization time (Table 1, Entries 1-6)

The ${ }^{1} \mathrm{H}-\mathrm{NMR}$ has shown the detailed information about the resultant polymers in various polymerization times when the monomer/catalyst ratio was 40 . The peaks between 6.1-6.5 ppm which assigned to the double bond proton in the norbornadiene unit as the chain end group became weaker gradually along with the polymerization time and the increase of $M_{\mathrm{n}}$. 


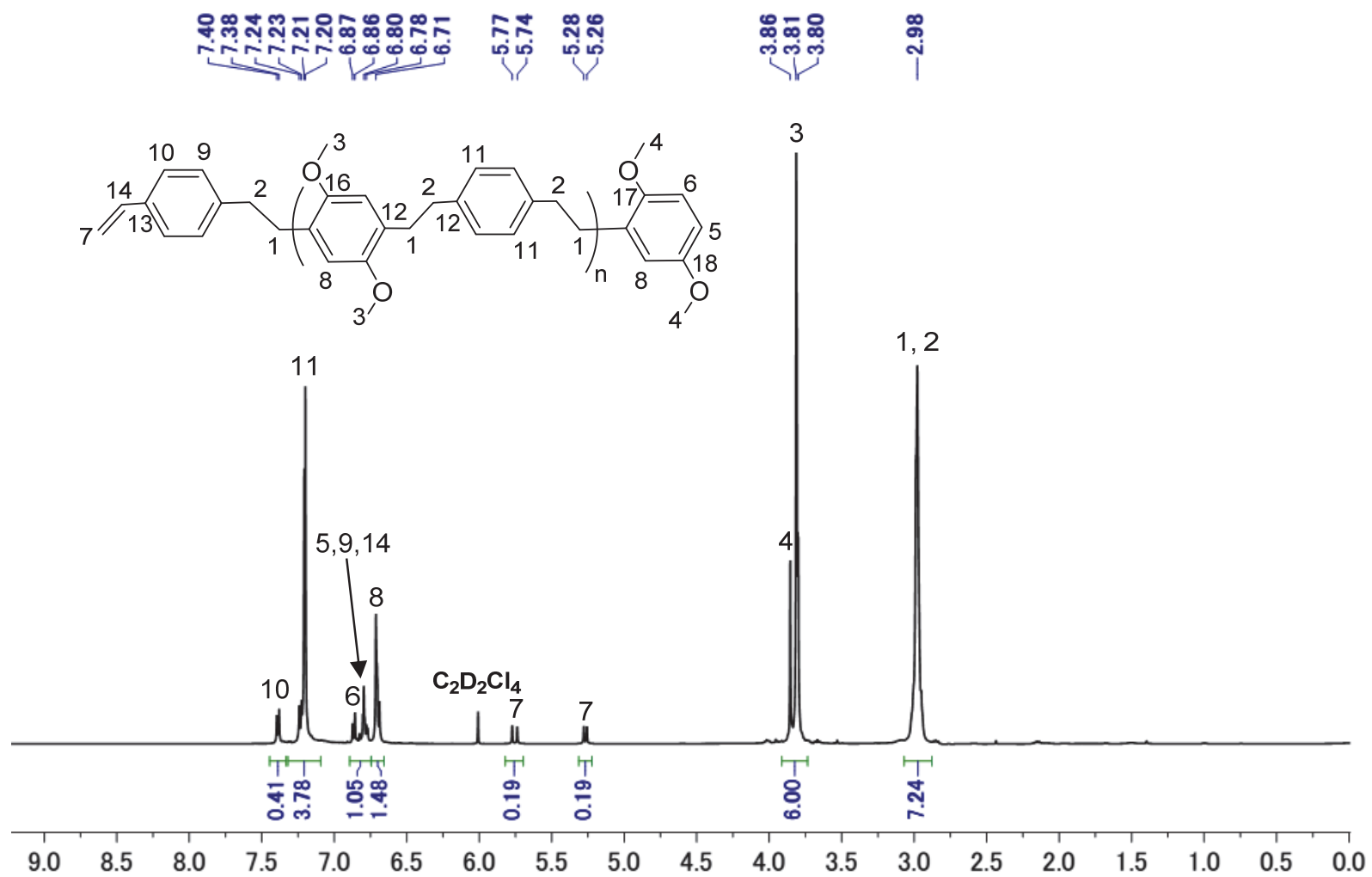

Figure S7. ${ }^{1} \mathrm{H}$ NMR spectrum $\left(500 \mathrm{MHz}, \mathrm{C}_{2} \mathrm{D}_{2} \mathrm{Cl}_{4}, 120{ }^{\circ} \mathrm{C}\right)$ of copoly(1,4-dimethoxybenzene/1,4divinylbenzene) (Table 2, Entry 3 )
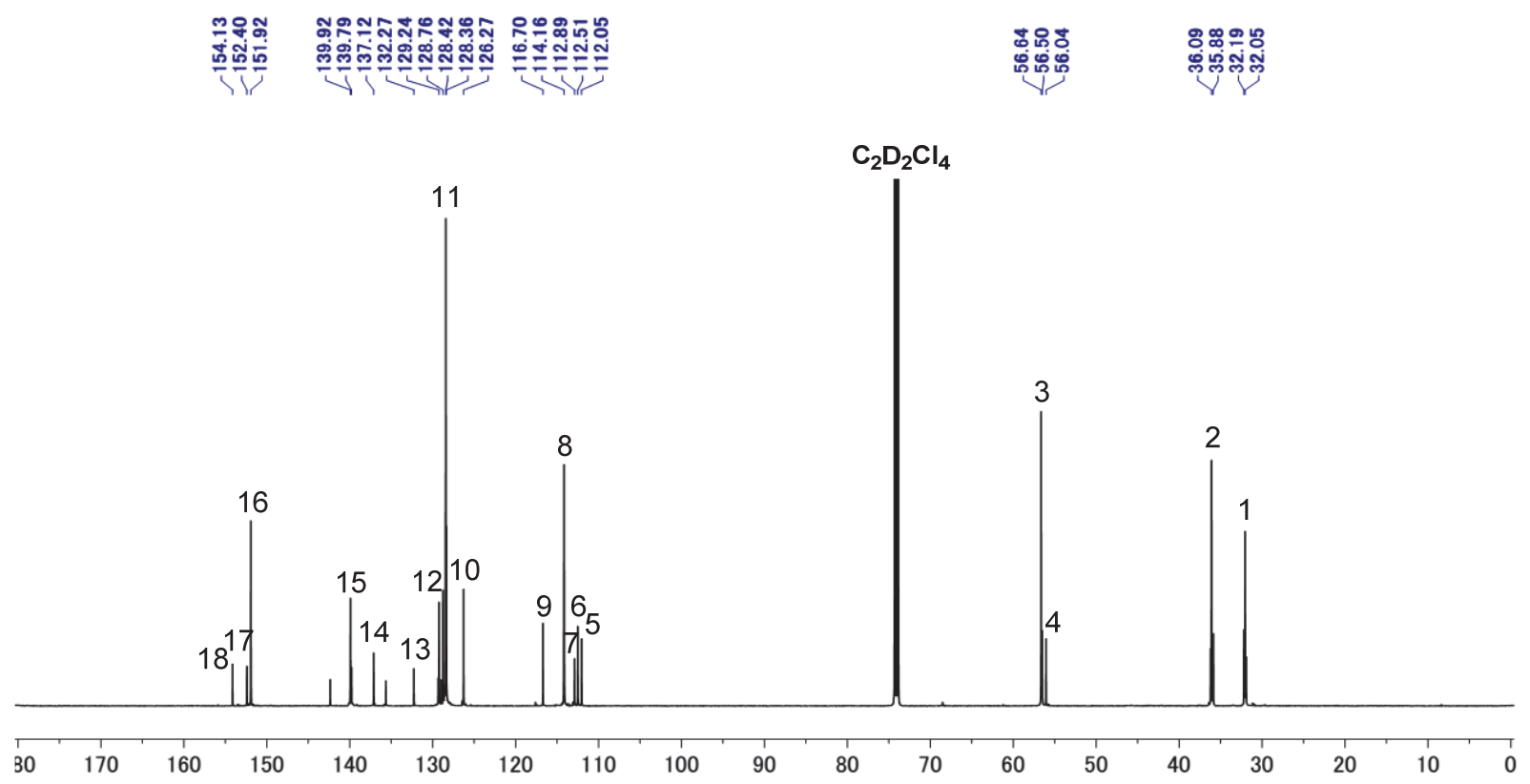

Figure S8. ${ }^{13} \mathrm{C}$ NMR spectrum $\left(125 \mathrm{MHz}, \mathrm{C}_{2} \mathrm{D}_{2} \mathrm{Cl}_{4}, 120{ }^{\circ} \mathrm{C}\right)$ of copoly(1,4-dimethoxybenzene/1,4divinylbenzene) (Table 2, Entry 3 ) 


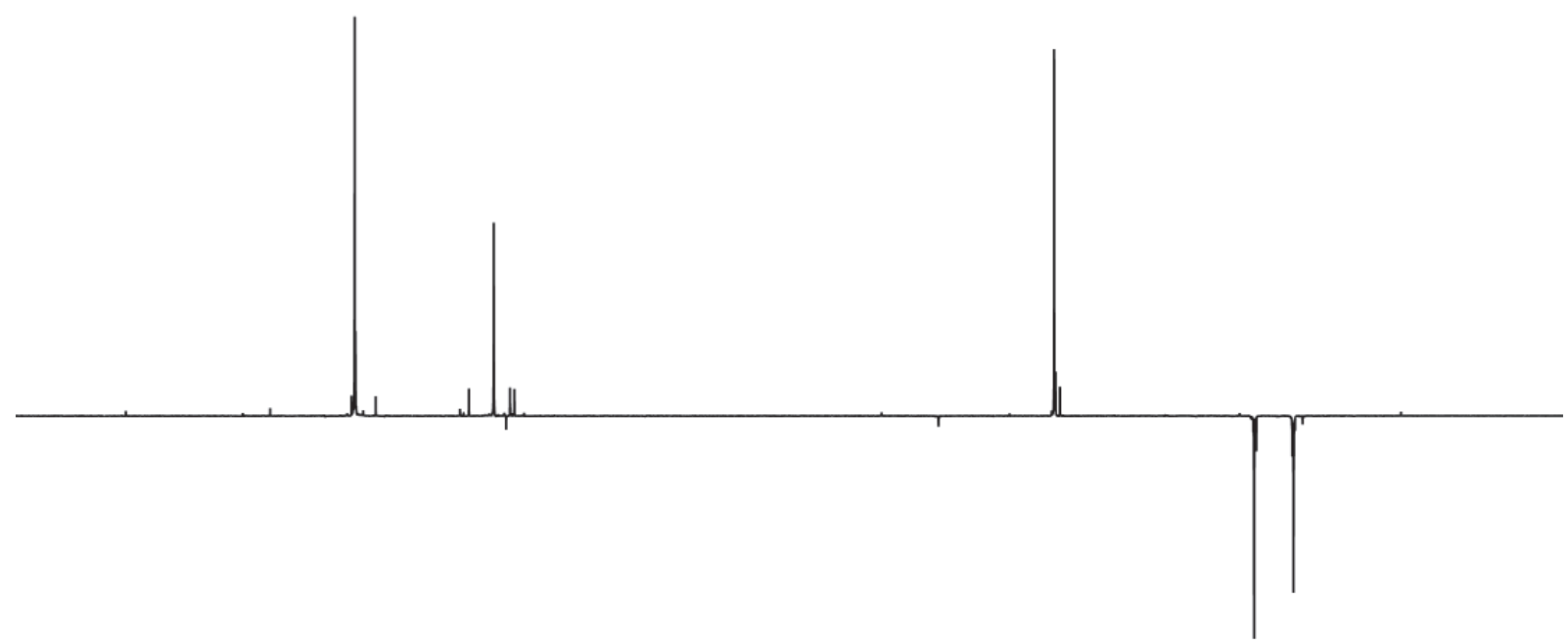

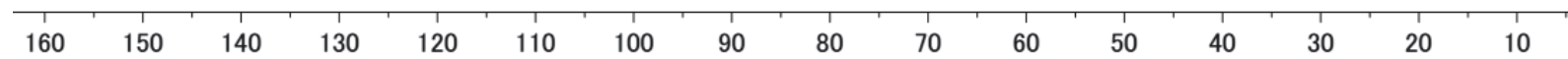

Figure S9. DEPT135- ${ }^{13} \mathrm{C}$ spectrum $\left(125 \mathrm{MHz}, \mathrm{C}_{2} \mathrm{D}_{2} \mathrm{Cl}_{4}, 120{ }^{\circ} \mathrm{C}\right)$ of copoly(1,4-dimethoxybenzene/1,4divinylbenzene) (Table 2, Entry 3)

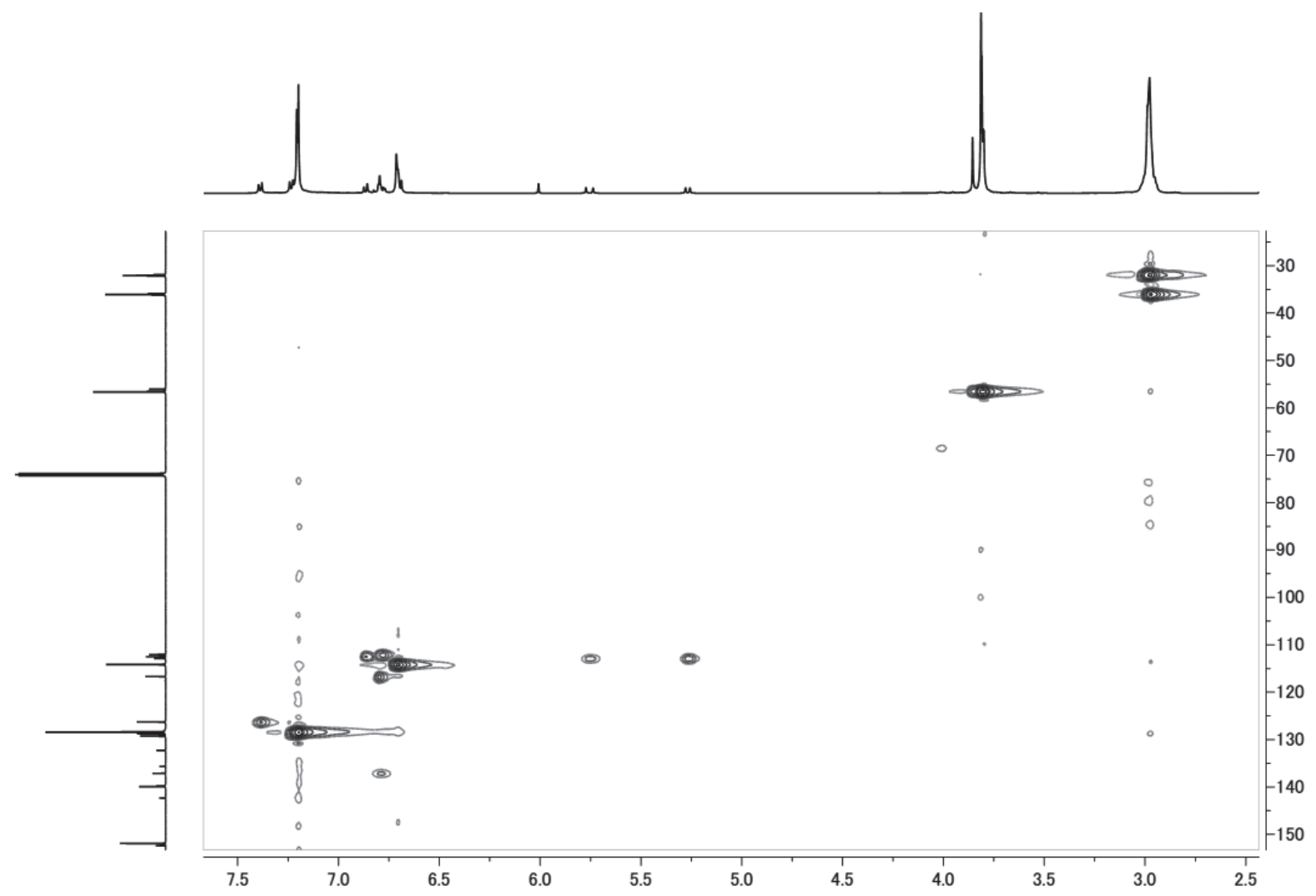

Figure 10. HSQC spectrum $\left(\mathrm{C}_{2} \mathrm{D}_{2} \mathrm{Cl}_{4}, 120{ }^{\circ} \mathrm{C}\right)$ of copoly(1,4-dimethoxybenzene/1,4-divinylbenzene) (Table 2, Entry 3) 


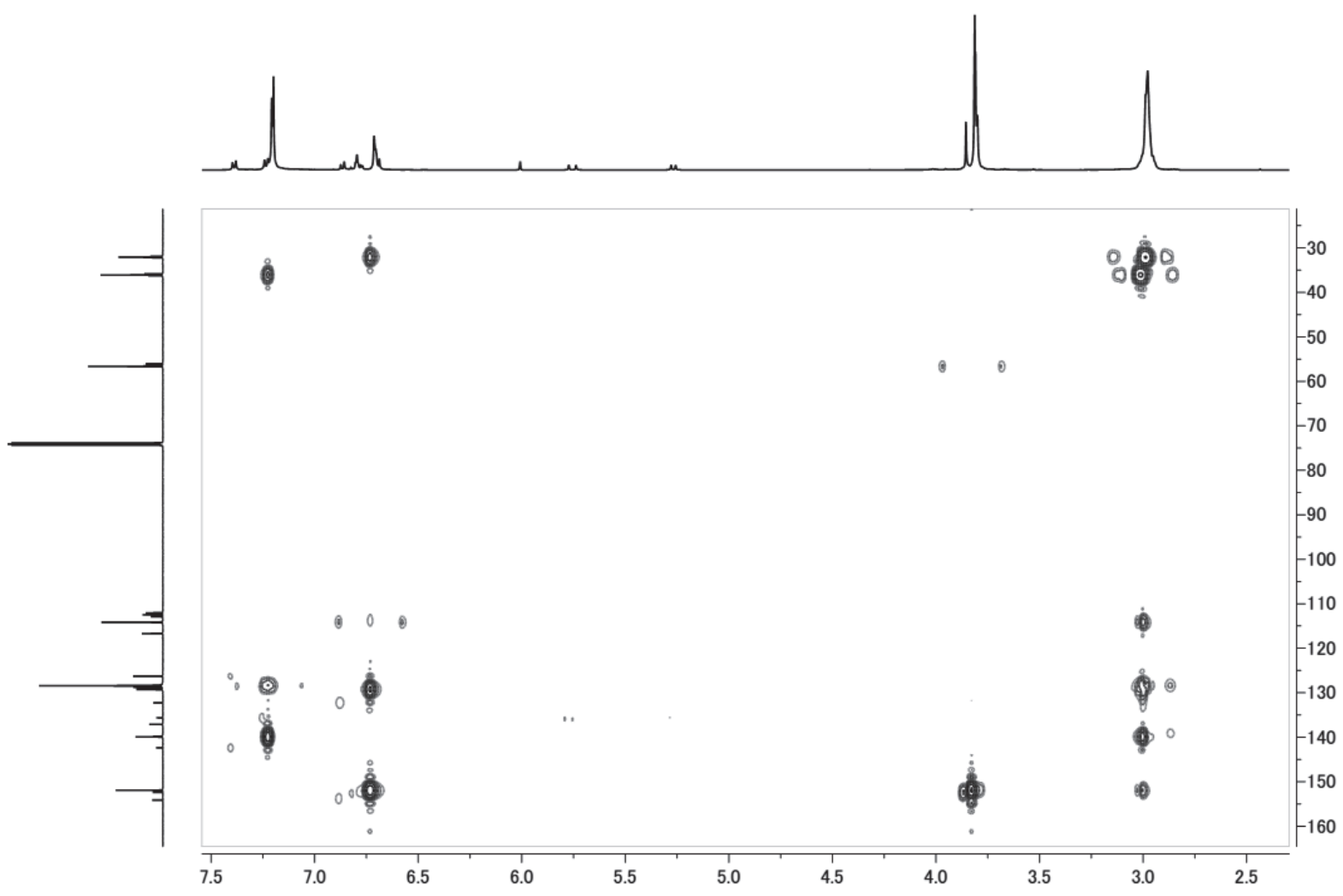

Figure S11. HMBC spectrum $\left(\mathrm{C}_{2} \mathrm{D}_{2} \mathrm{Cl}_{4}, 120^{\circ} \mathrm{C}\right)$ of copoly(1,4-dimethoxybenzene/1,4-divinylbenzene) (Table 2, Entry 3) 


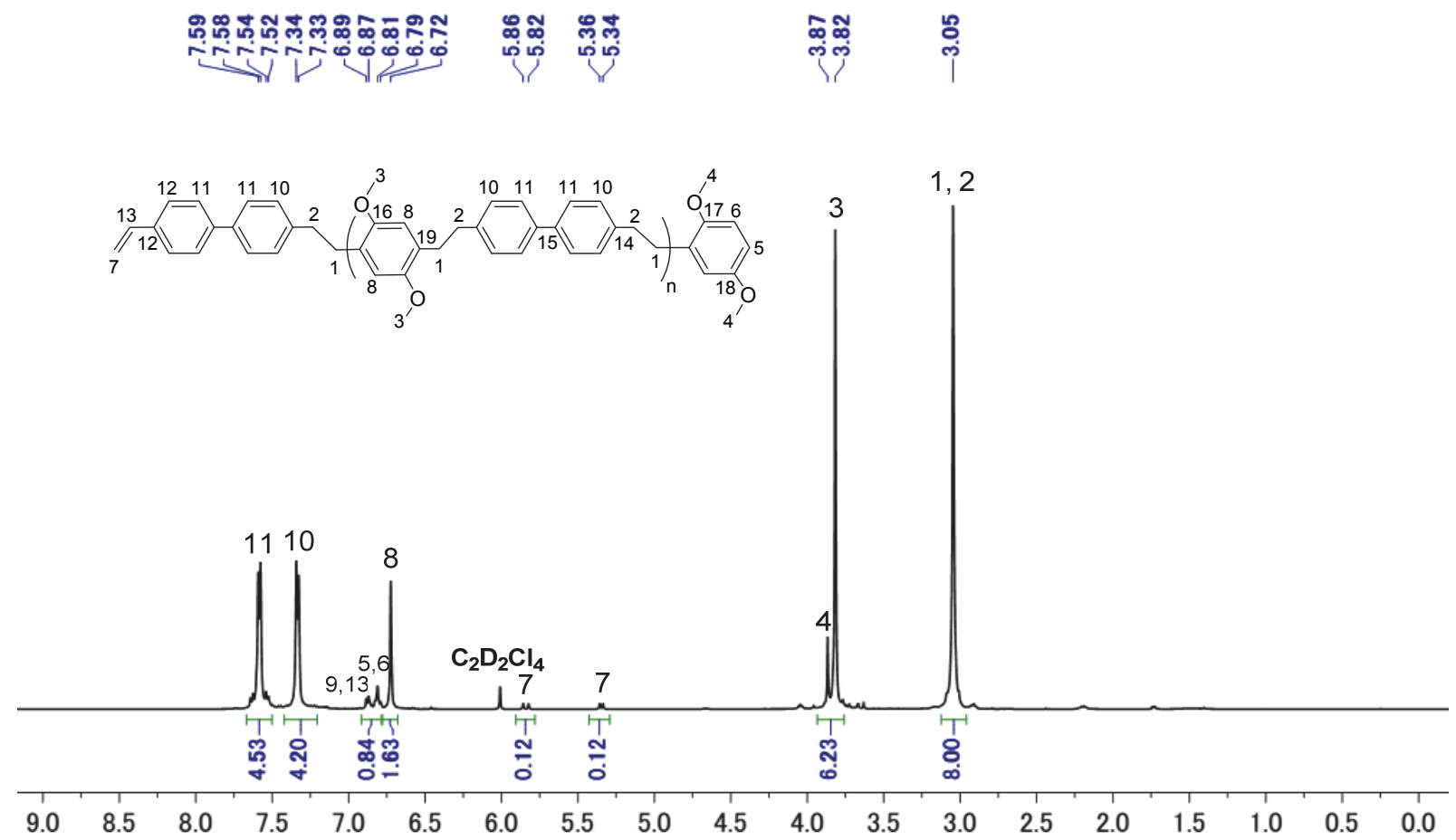

Figure S12. ${ }^{1} \mathrm{H}$ NMR spectrum $\left(500 \mathrm{MHz}, \mathrm{C}_{2} \mathrm{D}_{2} \mathrm{Cl}_{4}, 120{ }^{\circ} \mathrm{C}\right)$ of copoly(1,4-dimethoxybenzene/4,4'divinylbiphenyl)
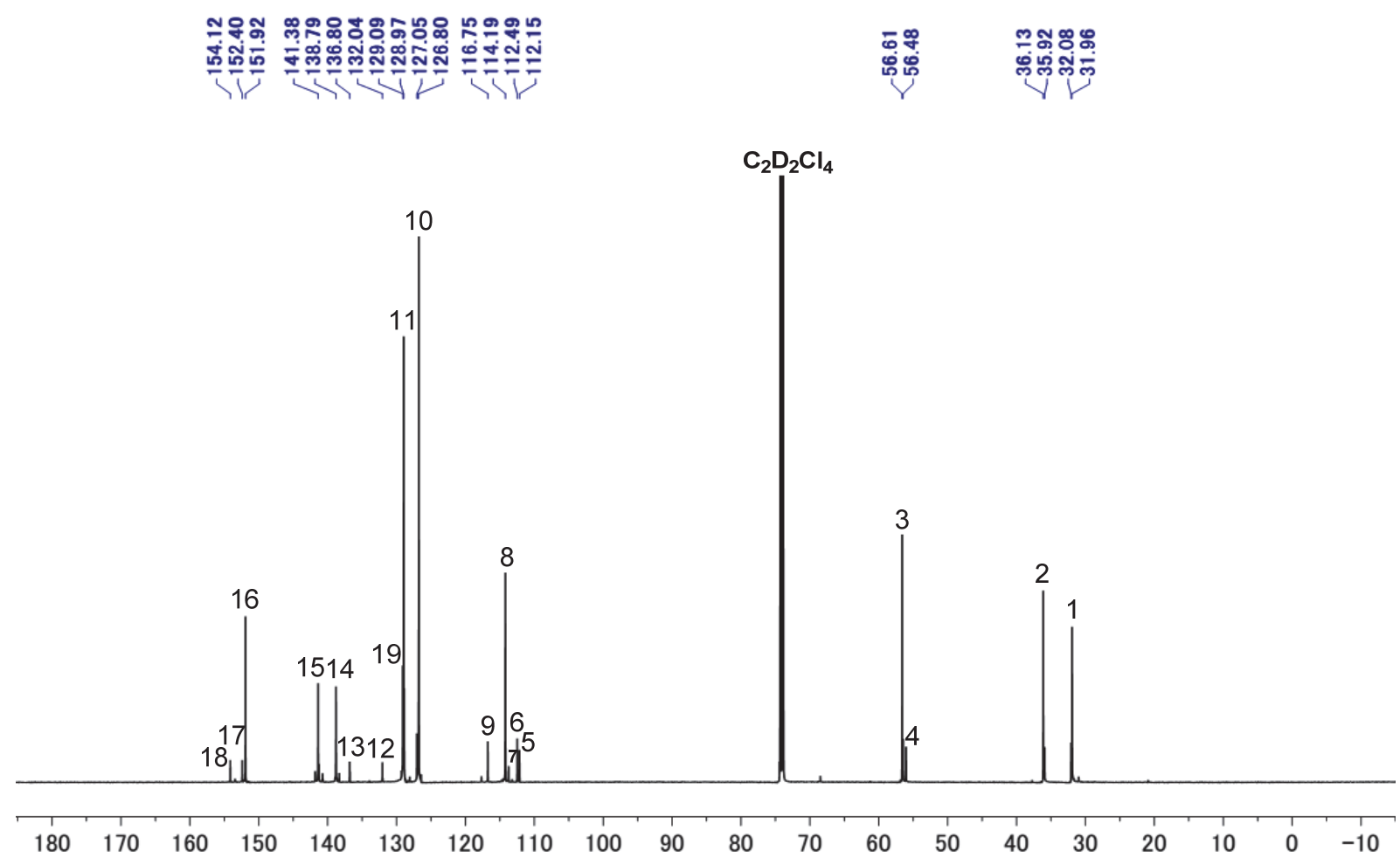

Figure S13. ${ }^{13} \mathrm{C}$ NMR spectrum $\left(125 \mathrm{MHz}, \mathrm{C}_{2} \mathrm{D}_{2} \mathrm{Cl}_{4}, 120{ }^{\circ} \mathrm{C}\right)$ of copoly(1,4-dimethoxybenzene/4,4'divinylbiphenyl) 


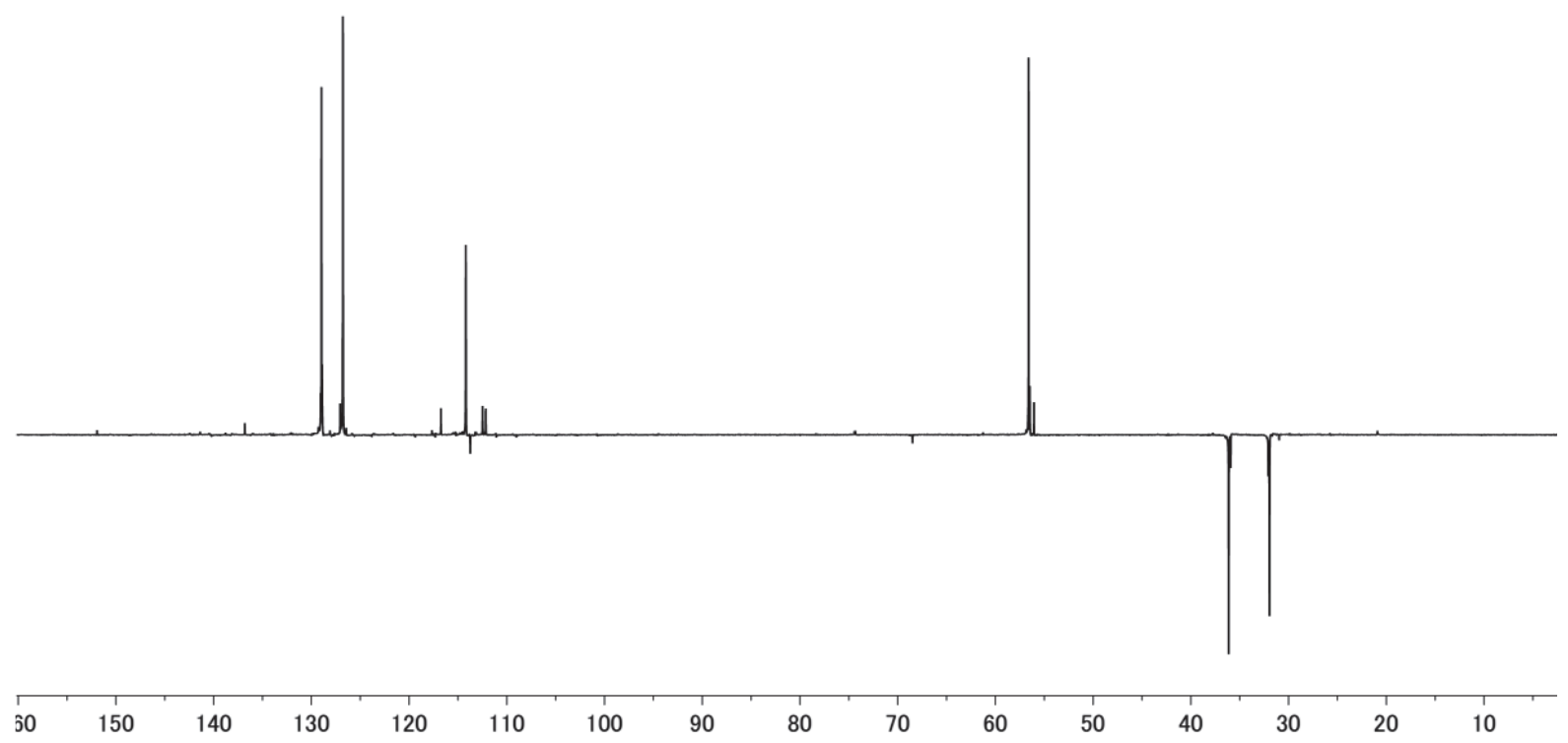

Figure S14. DEPT $135-{ }^{13} \mathrm{C}$ spectrum $\left(125 \mathrm{MHz}, \mathrm{C}_{2} \mathrm{D}_{2} \mathrm{Cl}_{4}, 120{ }^{\circ} \mathrm{C}\right)$ of copoly(1,4-dimethoxybenzene/4,4'divinylbiphenyl)

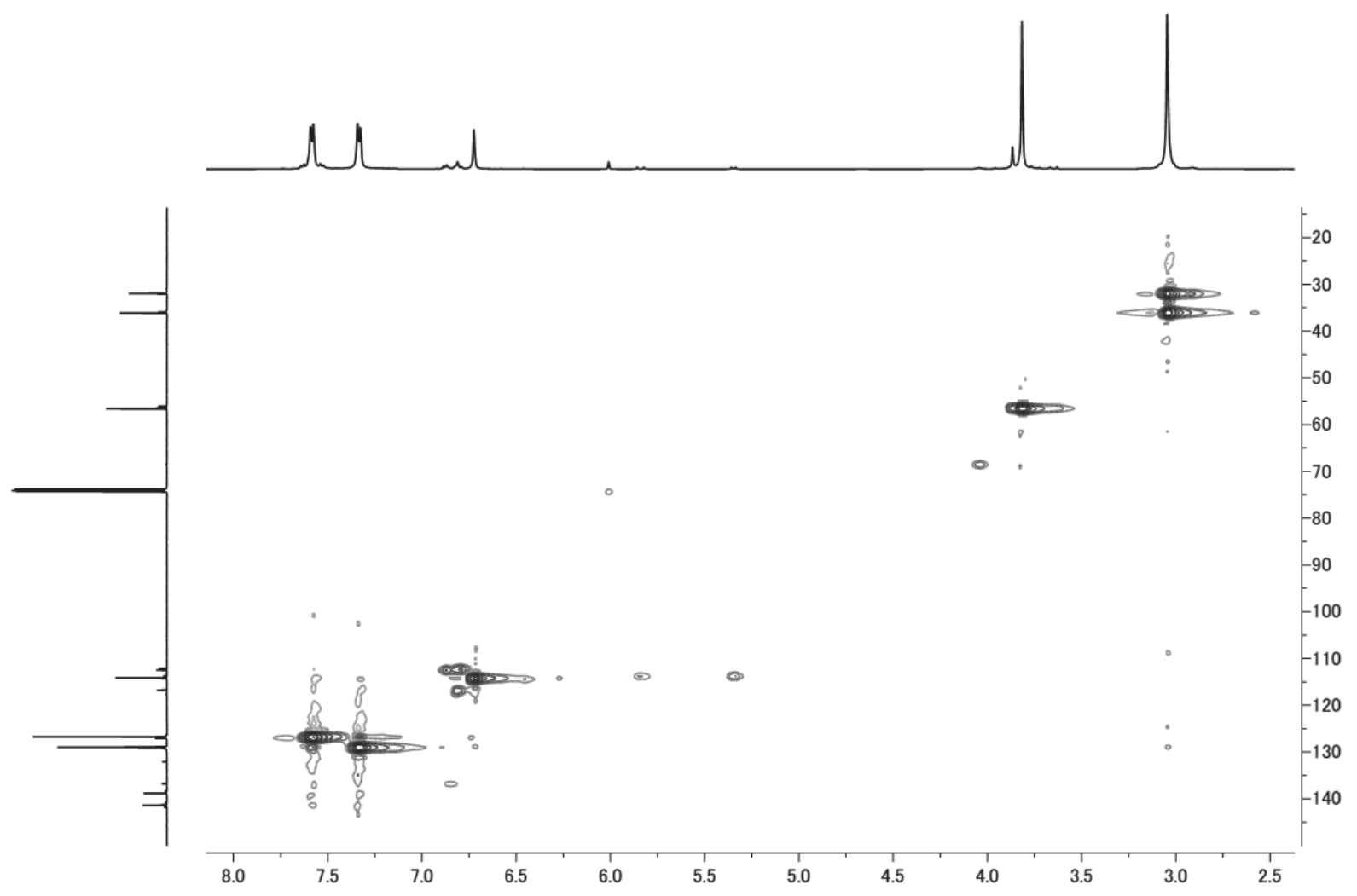

Figure S15. HSQC spectrum $\left(\mathrm{C}_{2} \mathrm{D}_{2} \mathrm{Cl}_{4}, 120^{\circ} \mathrm{C}\right)$ of copoly(1,4-dimethoxybenzene/4,4'-divinylbiphenyl) 


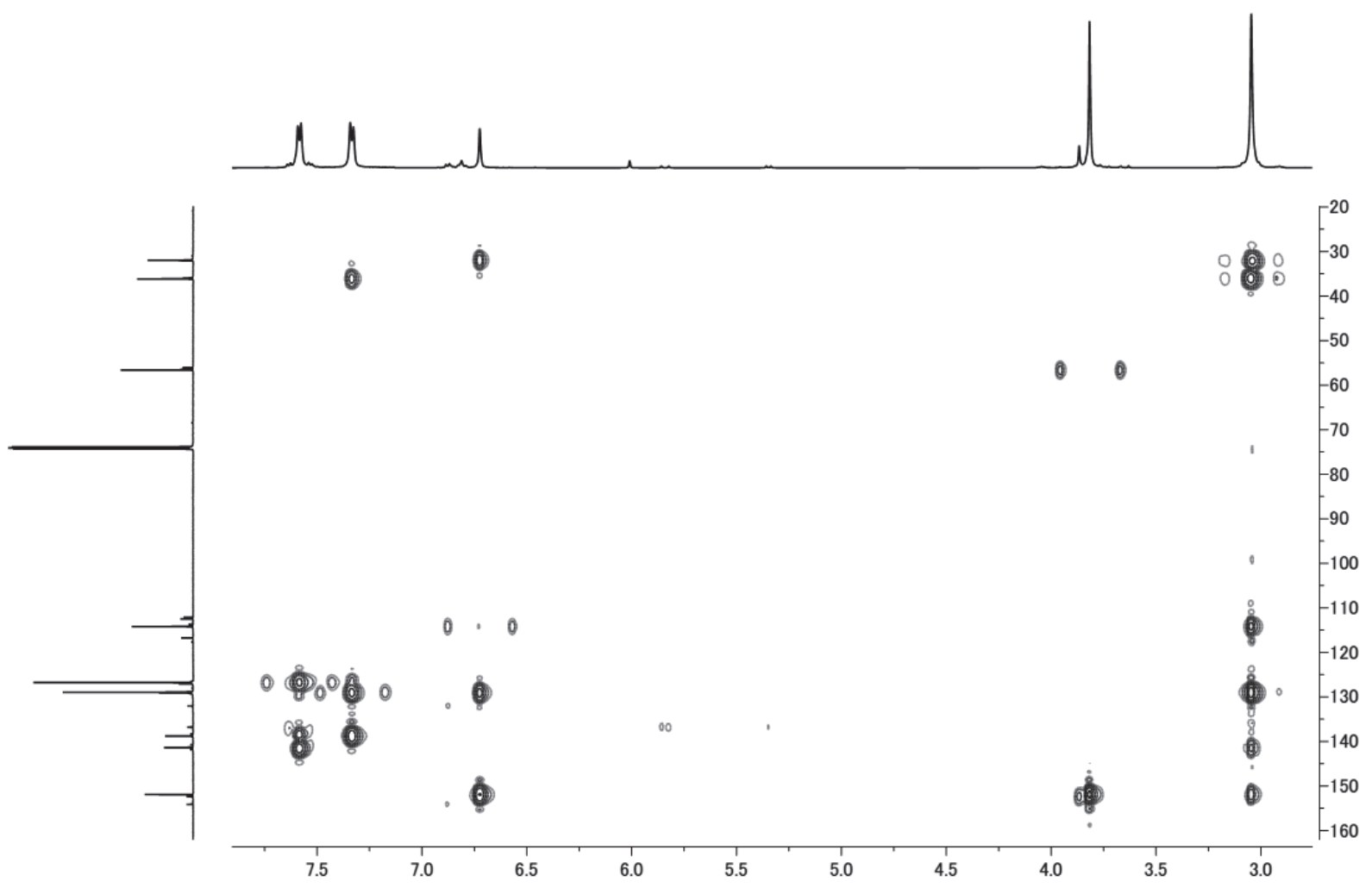

Figure S16. HMBC spectrum $\left(\mathrm{C}_{2} \mathrm{D}_{2} \mathrm{Cl}_{4}, 120^{\circ} \mathrm{C}\right)$ of copoly(1,4-dimethoxybenzene/4,4'-divinylbiphenyl) 


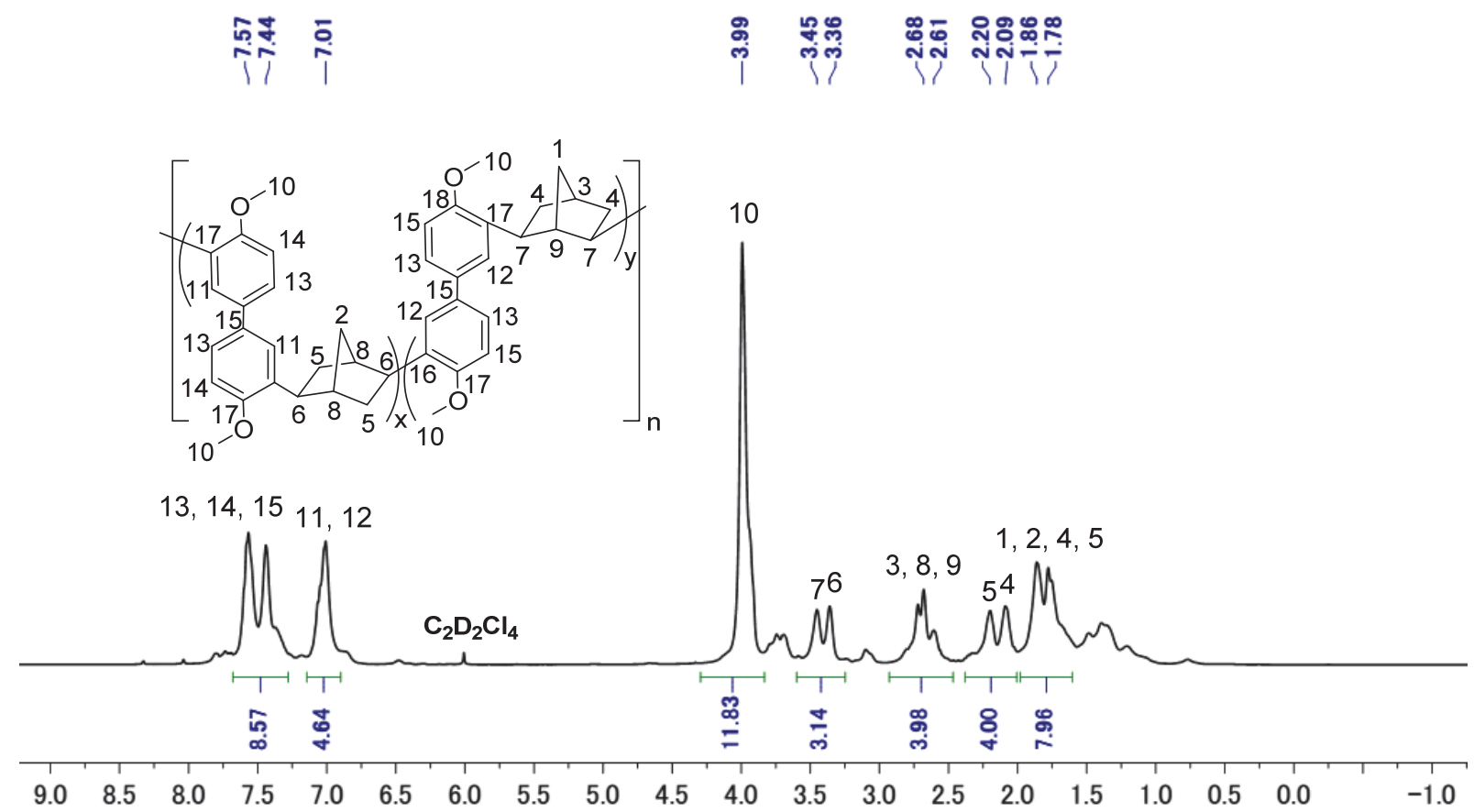

Figure S17. ${ }^{1} \mathrm{H}$ NMR spectrum $\left(500 \mathrm{MHz}, \mathrm{C}_{2} \mathrm{D}_{2} \mathrm{Cl}_{4}, 120{ }^{\circ} \mathrm{C}\right)$ of copoly(4,4'-dimethoxybiphenyl/ norbornadiene)
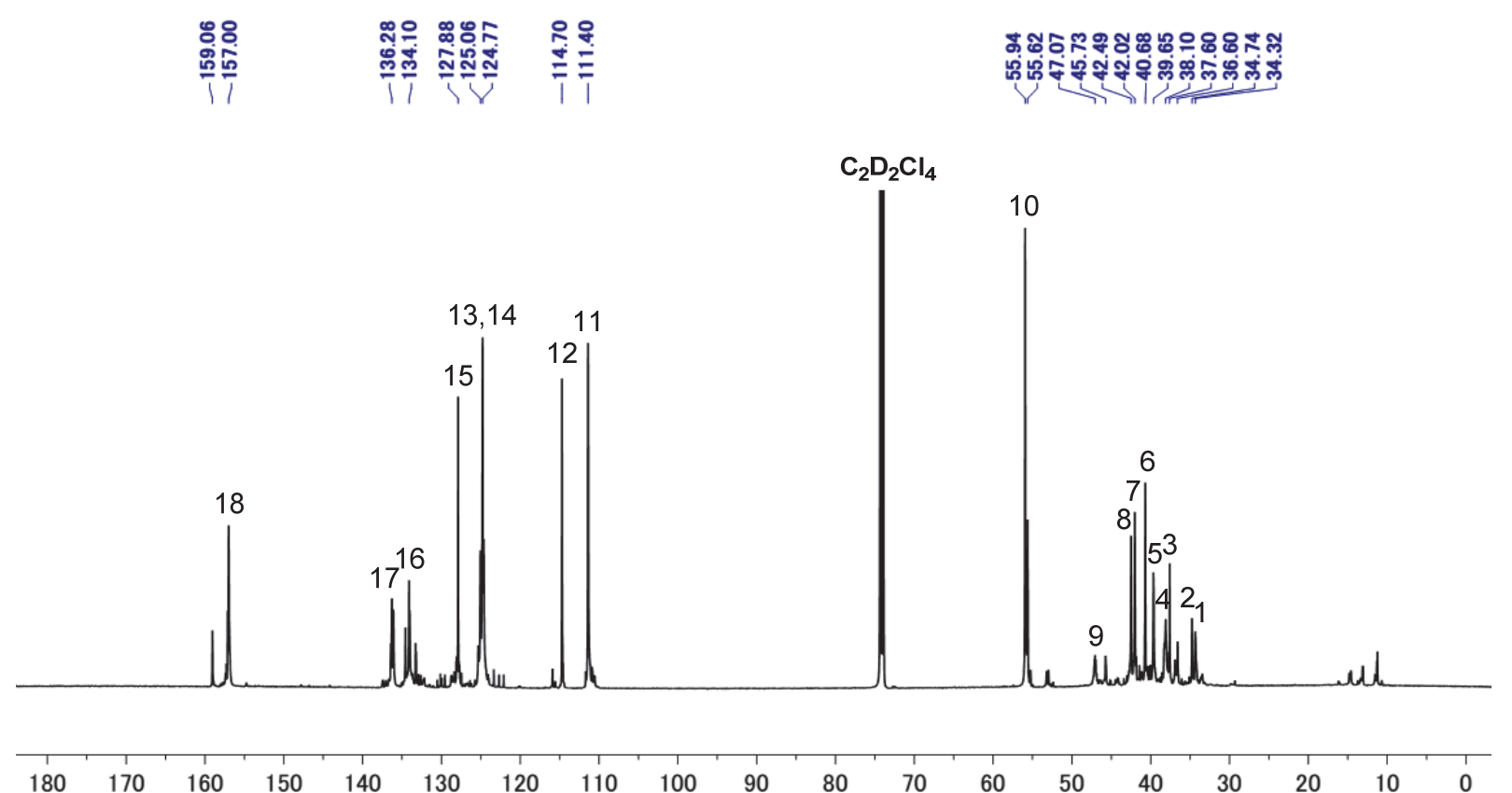

Figure S18. ${ }^{13} \mathrm{C}$ NMR spectrum $\left(125 \mathrm{MHz}, \mathrm{C}_{2} \mathrm{D}_{2} \mathrm{Cl}_{4}, 120{ }^{\circ} \mathrm{C}\right)$ of copoly(4,4'-dimethoxybiphenyl/ norbornadiene) 


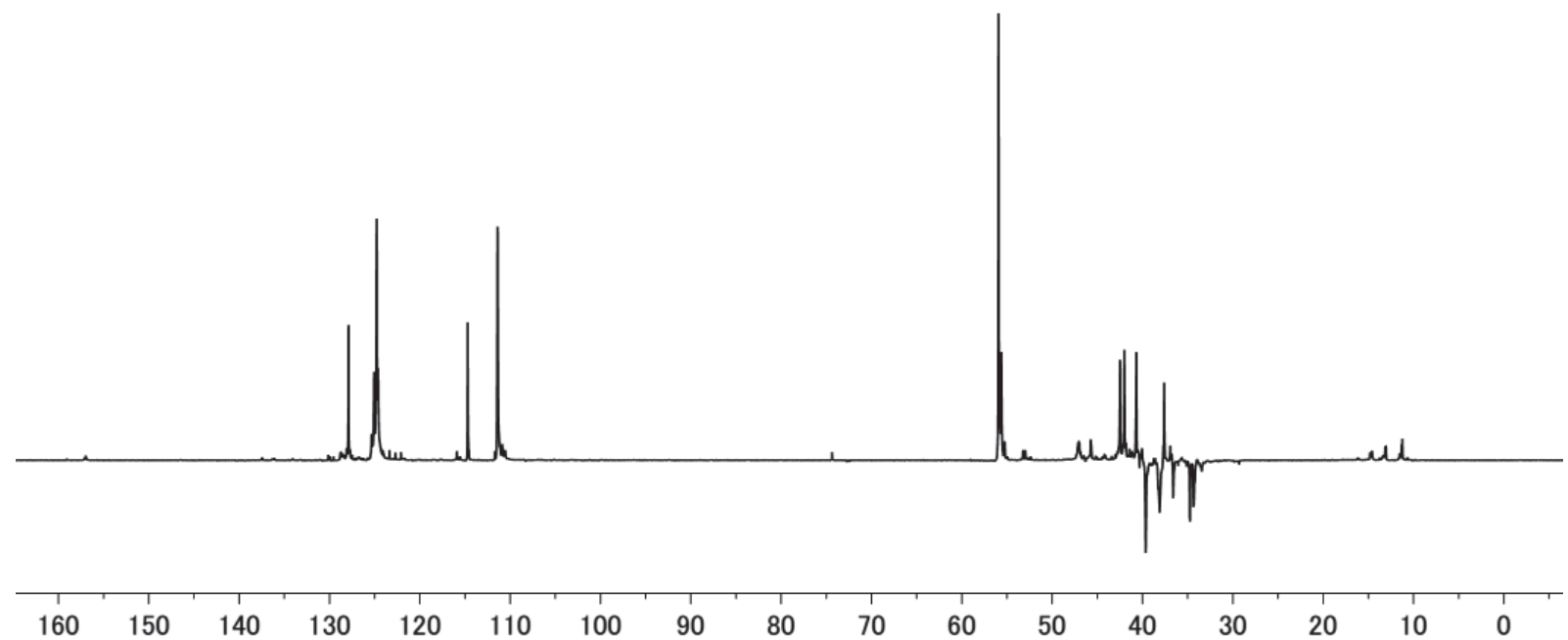

Figure S19. DEPT135- ${ }^{13} \mathrm{C}$ spectrum $\left(125 \mathrm{MHz}, \mathrm{C}_{2} \mathrm{D}_{2} \mathrm{Cl}_{4}, 120{ }^{\circ} \mathrm{C}\right)$ of copoly(4,4'-dimethoxybiphenyl/ norbornadiene)

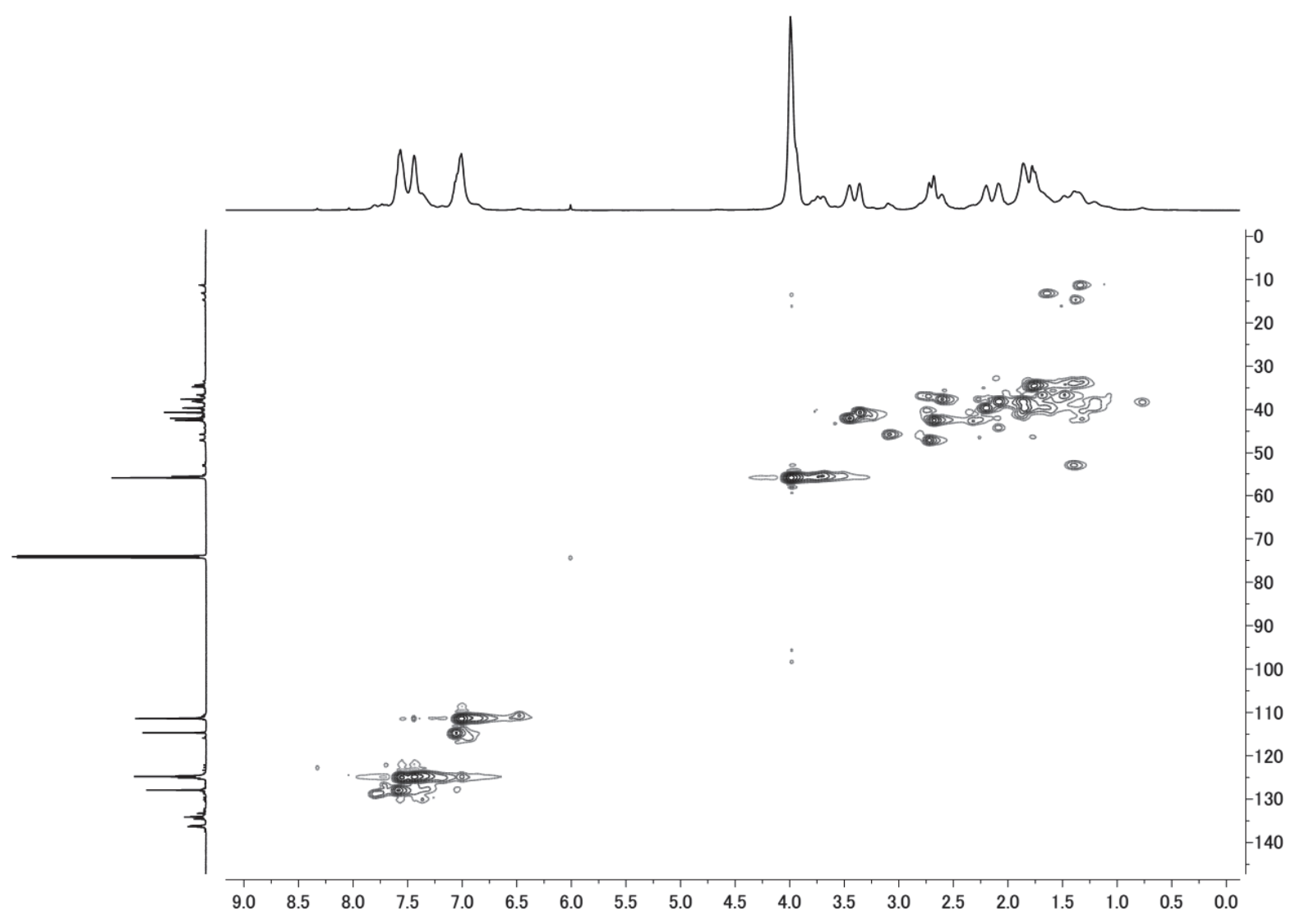

Figure S20. HSQC spectrum $\left(\mathrm{C}_{2} \mathrm{D}_{2} \mathrm{Cl}_{4}, 120^{\circ} \mathrm{C}\right)$ of copoly $\left(4,4^{\prime}\right.$-dimethoxybiphenyl/norbornadiene) 


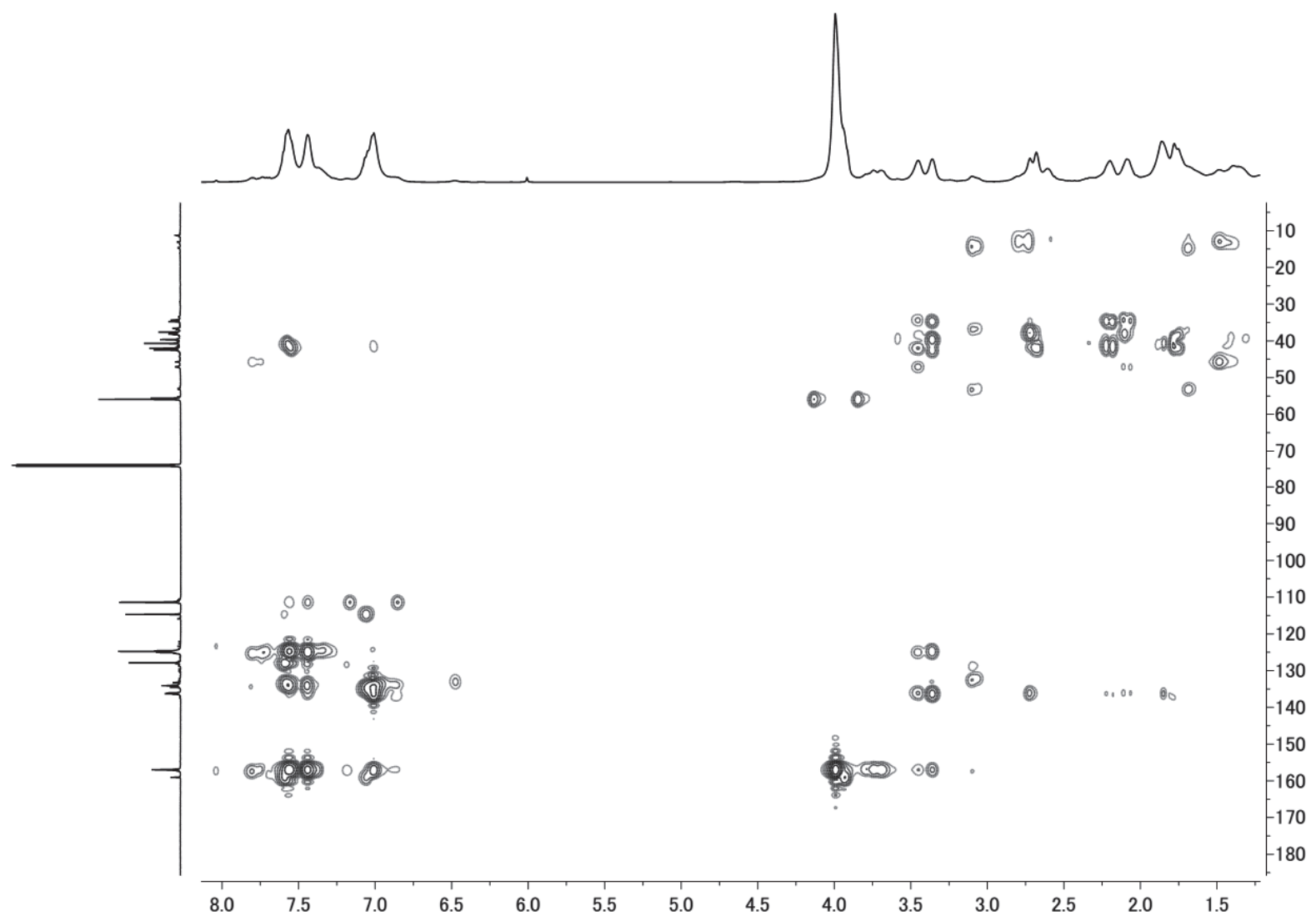

Figure S21. HMBC spectrum $\left(\mathrm{C}_{2} \mathrm{D}_{2} \mathrm{Cl}_{4}, 120^{\circ} \mathrm{C}\right)$ of copoly $\left(4,4^{\prime}\right.$-dimethoxybiphenyl/norbornadiene) 


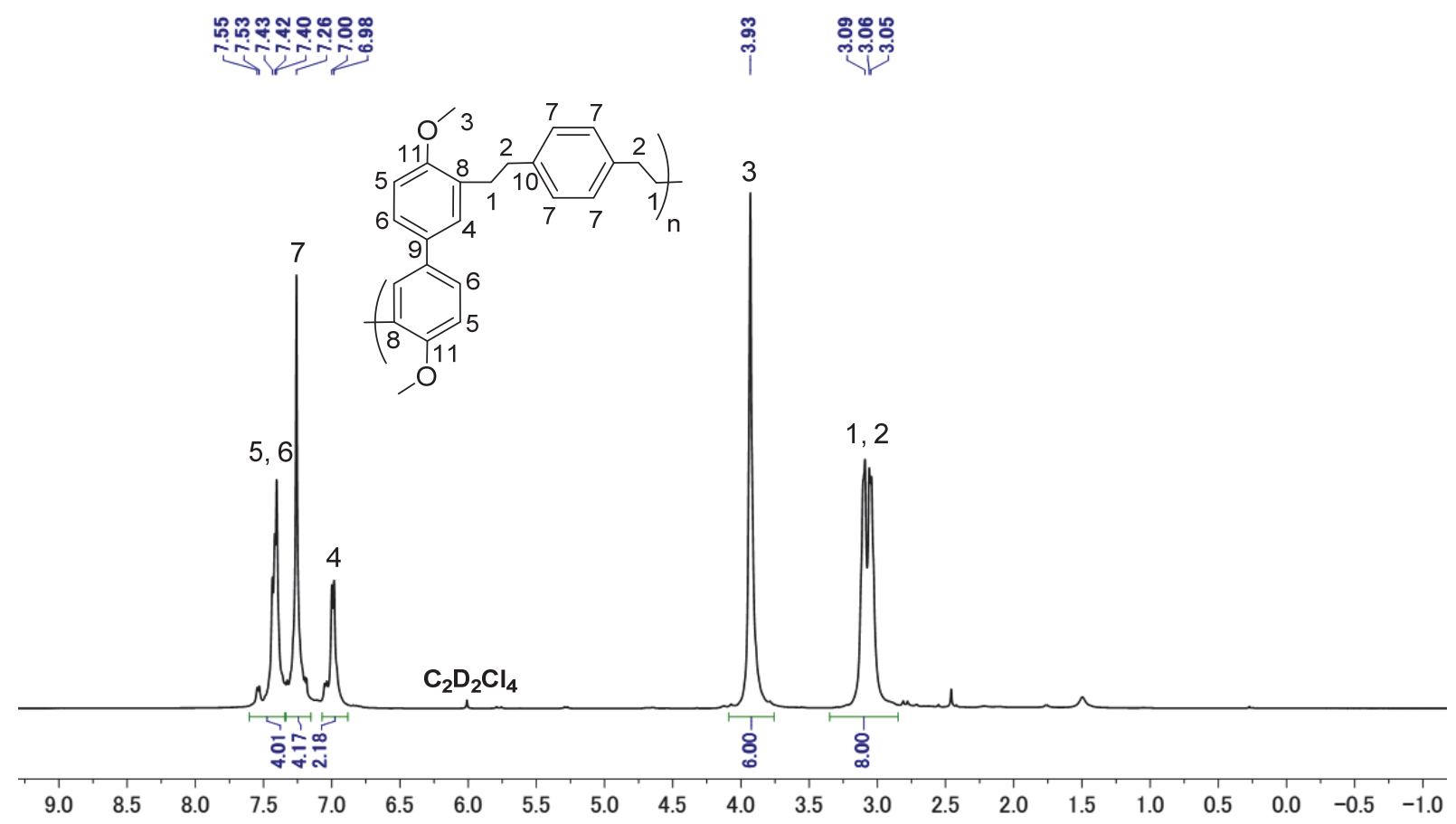

Figure S22. ${ }^{1} \mathrm{H}$ NMR spectrum $\left(500 \mathrm{MHz}, \mathrm{C}_{2} \mathrm{D}_{2} \mathrm{Cl}_{4}, 120{ }^{\circ} \mathrm{C}\right)$ of copoly $\left(4,4^{\prime}\right.$-dimethoxybiphenyl/1,4divinylbenzene)
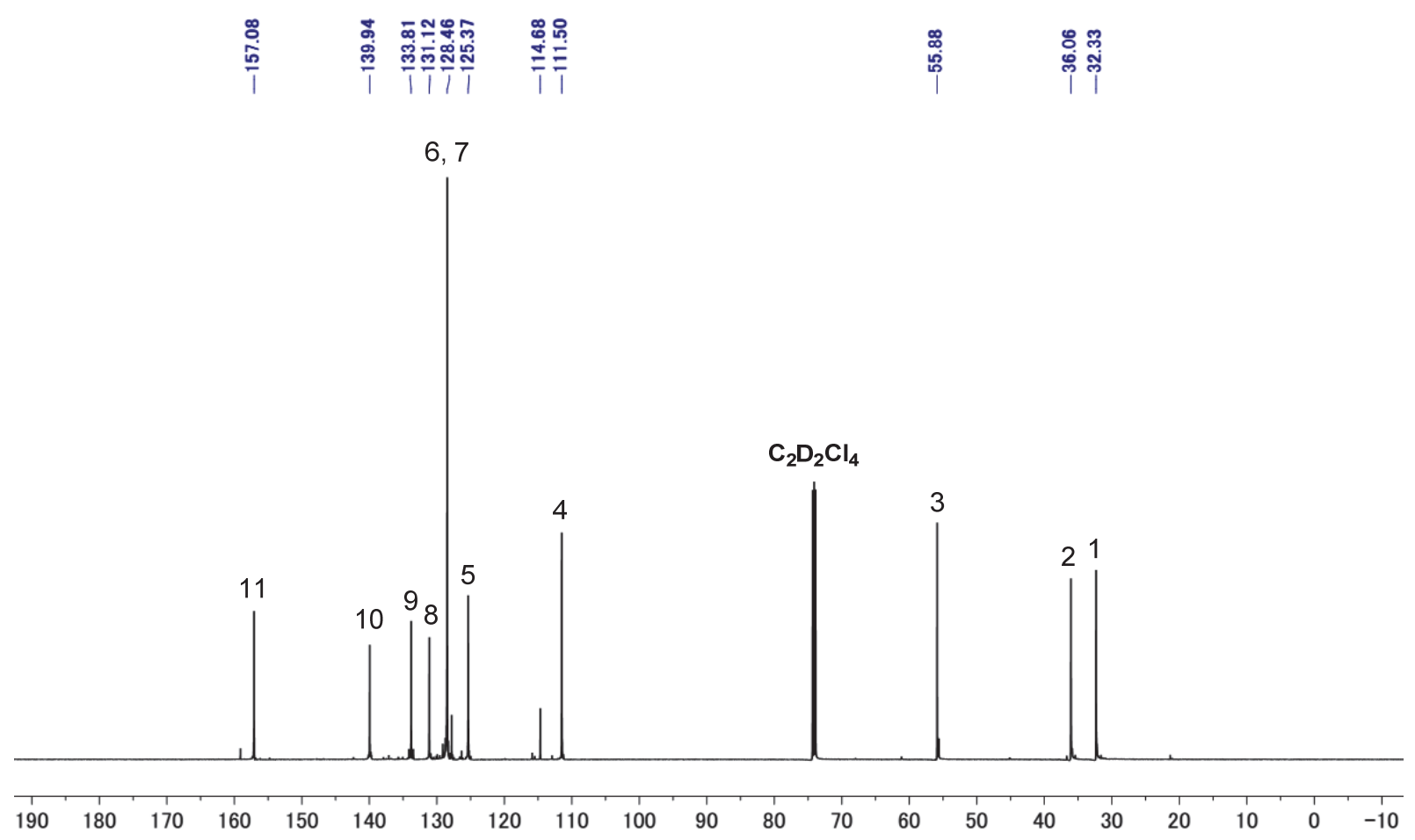

Figure S23. ${ }^{13} \mathrm{C}$ NMR spectrum $\left(125 \mathrm{MHz}, \mathrm{C}_{2} \mathrm{D}_{2} \mathrm{Cl}_{4}, 120{ }^{\circ} \mathrm{C}\right)$ of copoly $\left(4,4^{\prime}\right.$-dimethoxybiphenyl/1,4divinylbenzene) 


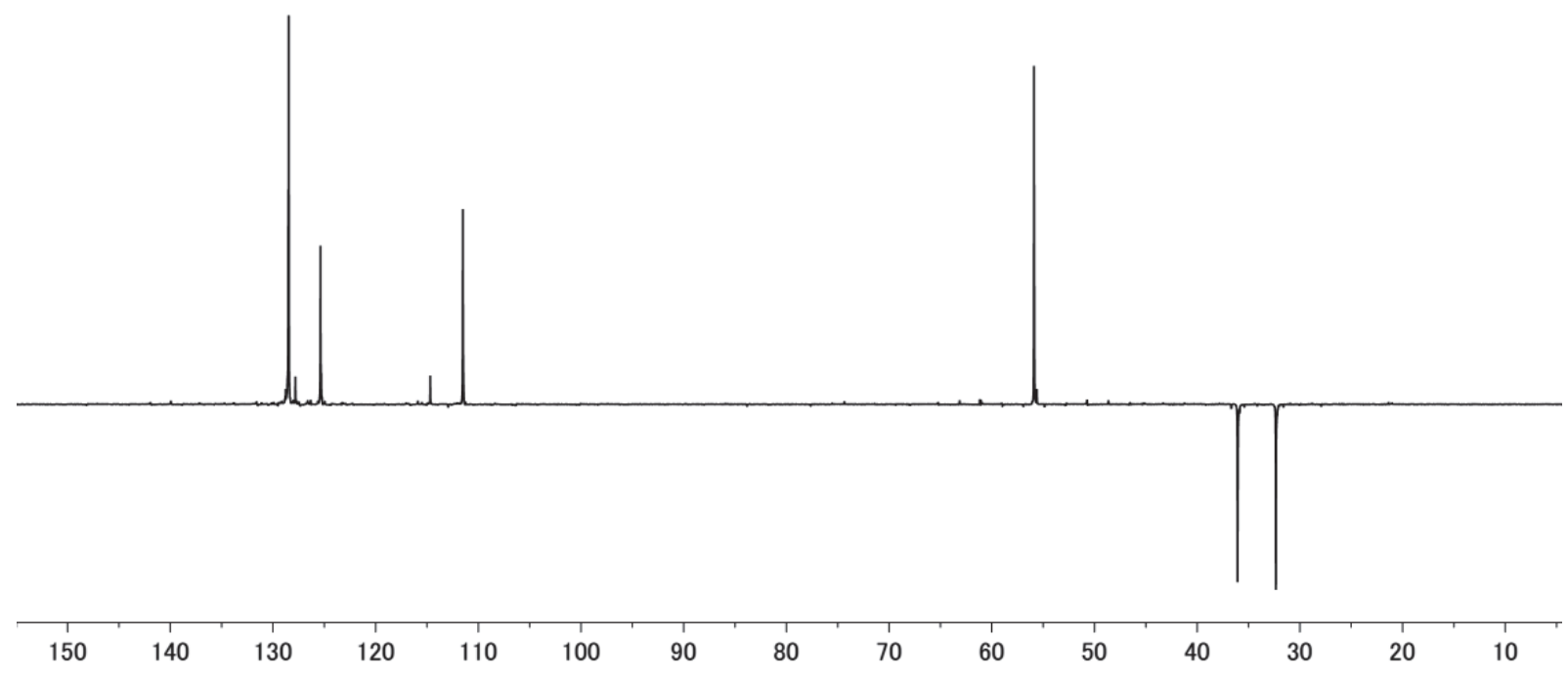

Figure S24. DEPT $135-{ }^{13} \mathrm{C}$ spectrum $\left(125 \mathrm{MHz}, \mathrm{C}_{2} \mathrm{D}_{2} \mathrm{Cl}_{4}, 120{ }^{\circ} \mathrm{C}\right)$ of copoly $\left(4,4^{\prime}\right.$-dimethoxybiphenyl/1,4divinylbenzene)

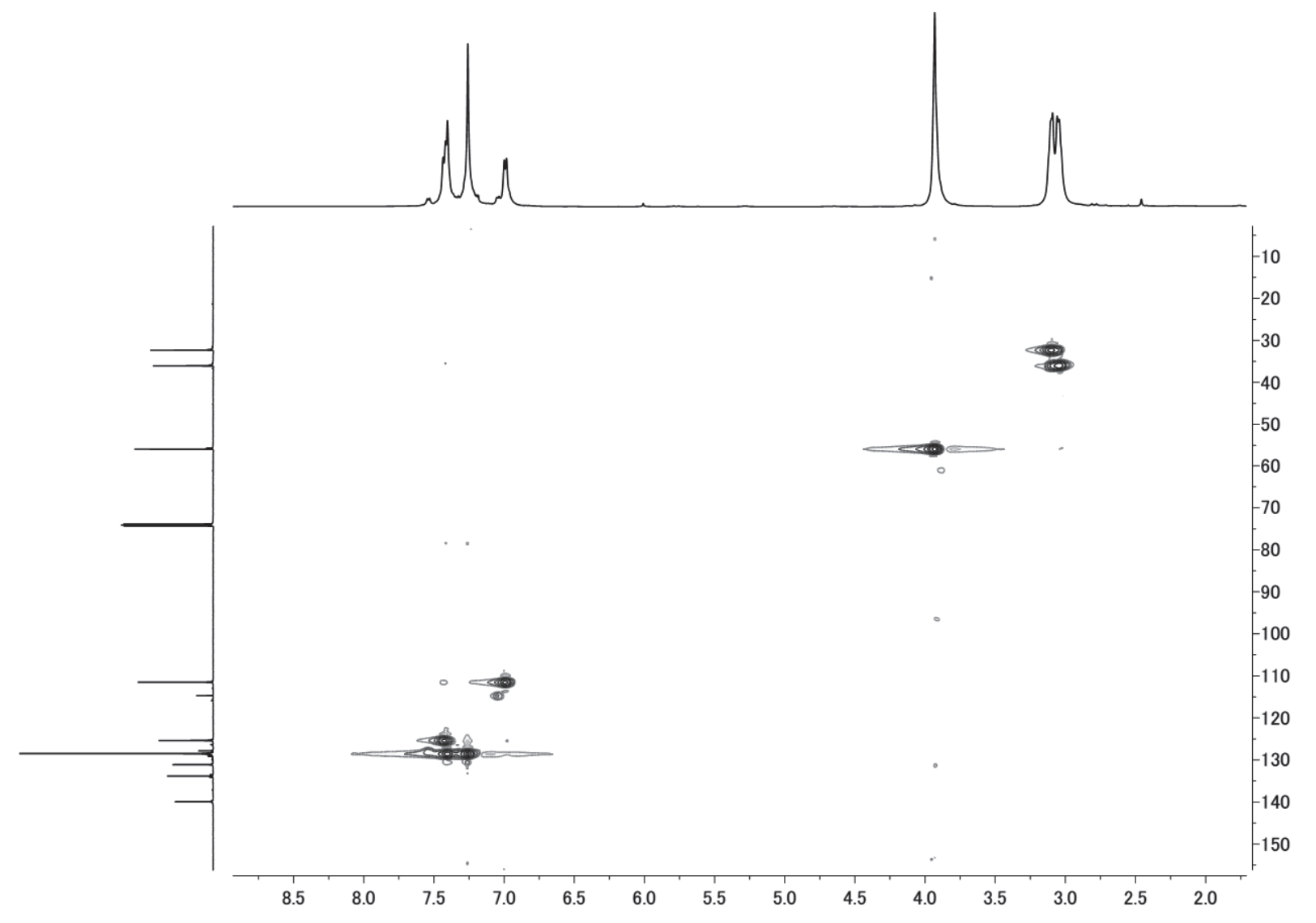

Figure S25. HSQC spectrum $\left(\mathrm{C}_{2} \mathrm{D}_{2} \mathrm{Cl}_{4}, 120^{\circ} \mathrm{C}\right)$ of copoly(4,4'-dimethoxybiphenyl/1,4-divinylbenzene) 


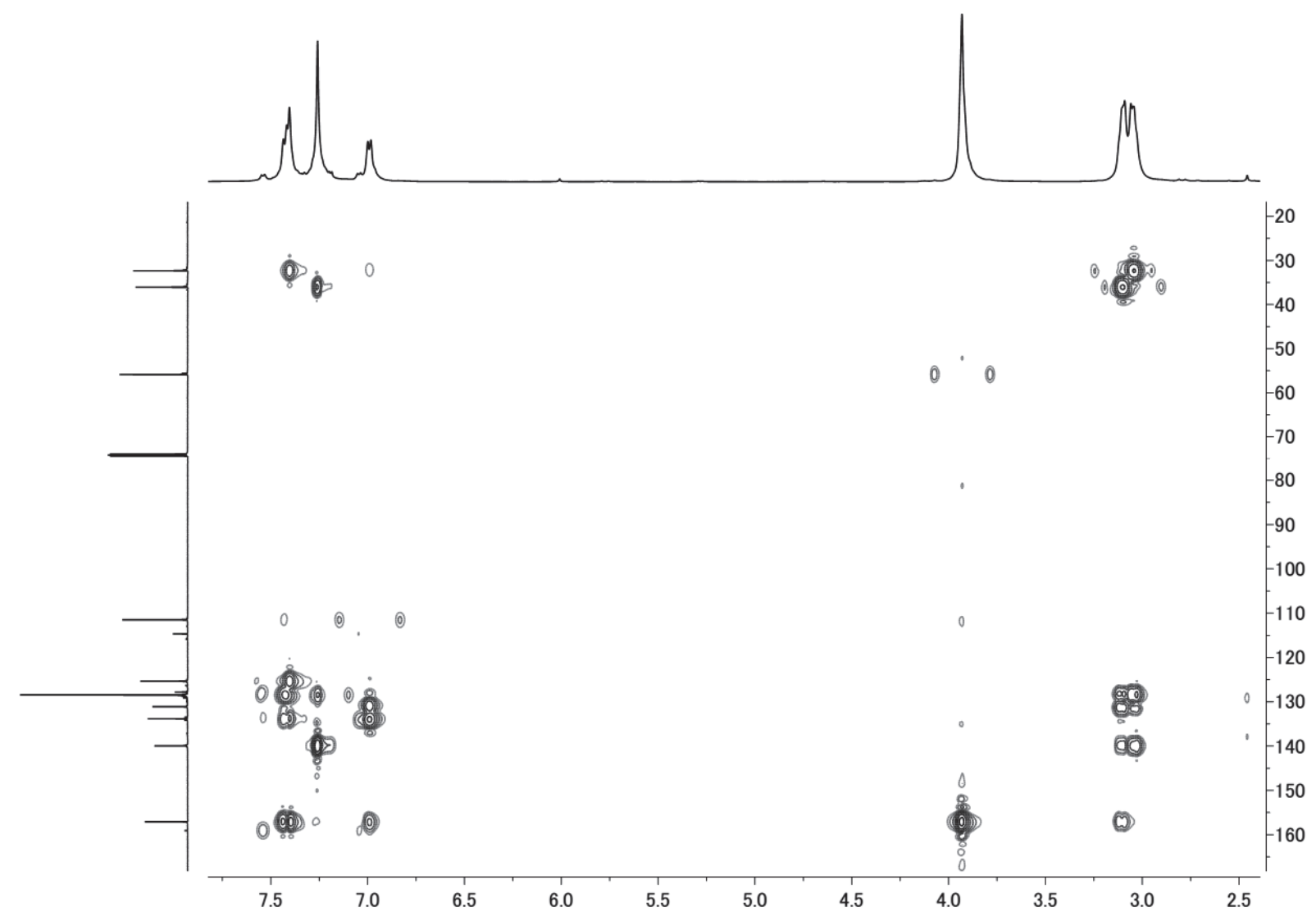

Figure S26. $\mathrm{HMBC}$ spectrum $\left(\mathrm{C}_{2} \mathrm{D}_{2} \mathrm{Cl}_{4}, 120^{\circ} \mathrm{C}\right)$ of copoly(4,4'-dimethoxybiphenyl/1,4-divinylbenzene) 

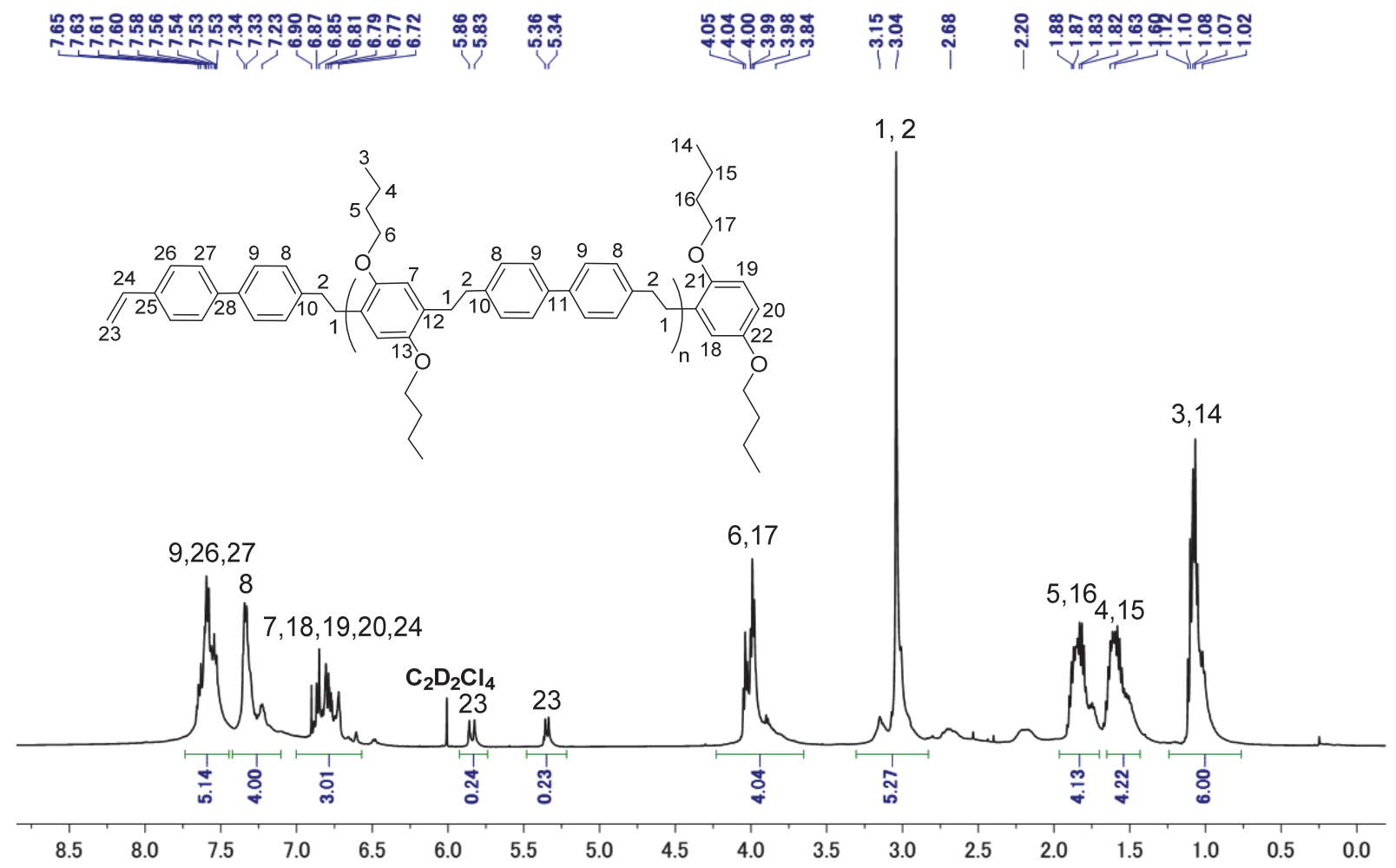

Figure S27. ${ }^{1} \mathrm{H}$ NMR spectrum $\left(500 \mathrm{MHz}, \mathrm{C}_{2} \mathrm{D}_{2} \mathrm{Cl}_{4}, 120{ }^{\circ} \mathrm{C}\right)$ of copoly(1,4-dibutoxybenzene/4,4'divinylbiphenyl)

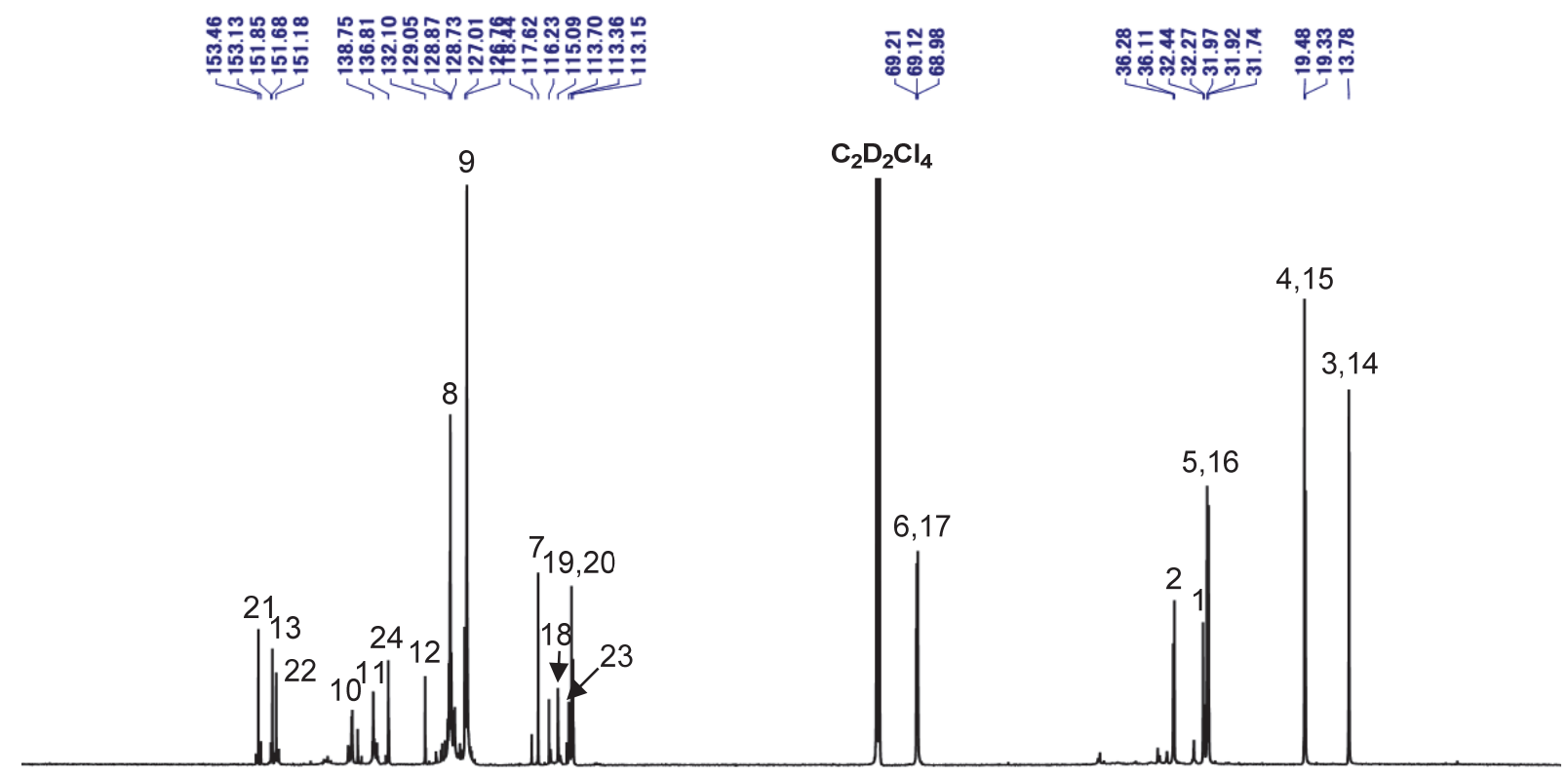

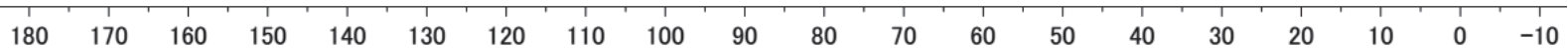

Figure S28. ${ }^{13} \mathrm{C}$ NMR spectrum $\left(125 \mathrm{MHz}, \mathrm{C}_{2} \mathrm{D}_{2} \mathrm{Cl}_{4}, 120{ }^{\circ} \mathrm{C}\right)$ of copoly(1,4-dibutoxybenzene/4,4'divinylbiphenyl) 


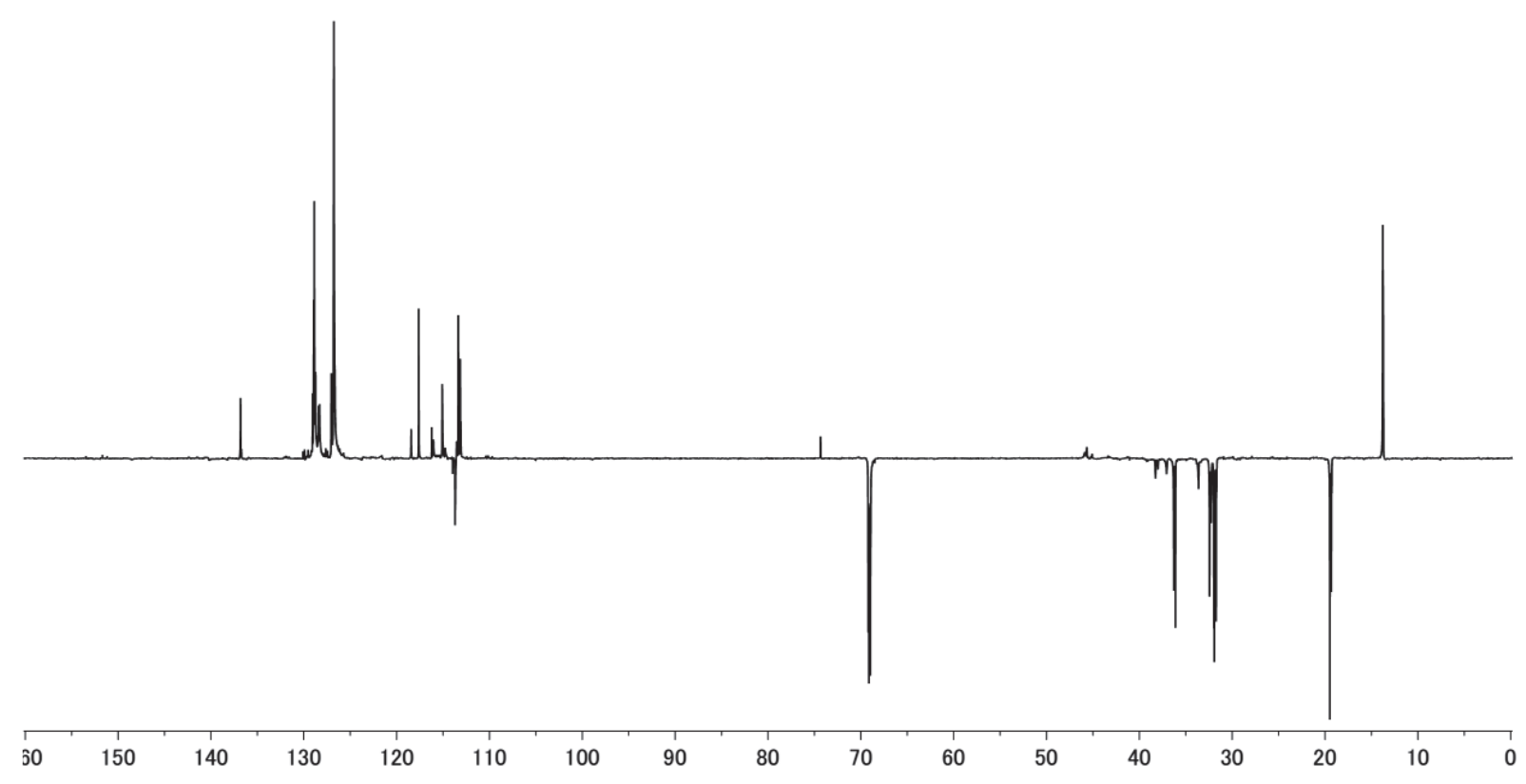

Figure S29. DEPT135- ${ }^{13} \mathrm{C}$ spectrum $\left(125 \mathrm{MHz}, \mathrm{C}_{2} \mathrm{D}_{2} \mathrm{Cl}_{4}, 120{ }^{\circ} \mathrm{C}\right)$ of copoly $(1,4$-dibutoxybenzene/4,4'divinylbiphenyl)

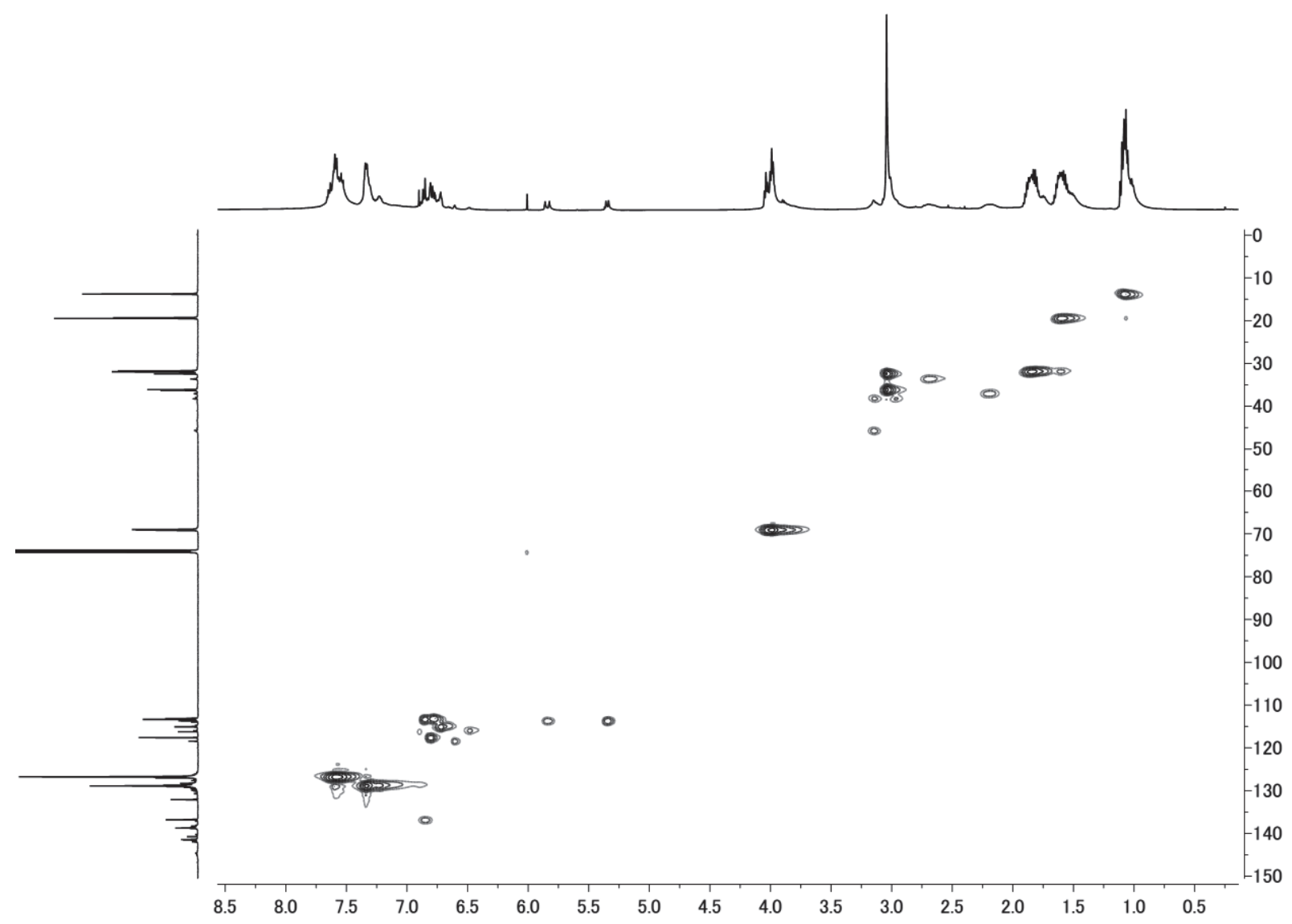

Figure S30. HSQC spectrum $\left(\mathrm{C}_{2} \mathrm{D}_{2} \mathrm{Cl}_{4}, 120^{\circ} \mathrm{C}\right)$ of copoly(1,4-dibutoxybenzene/4,4'-divinylbiphenyl) 


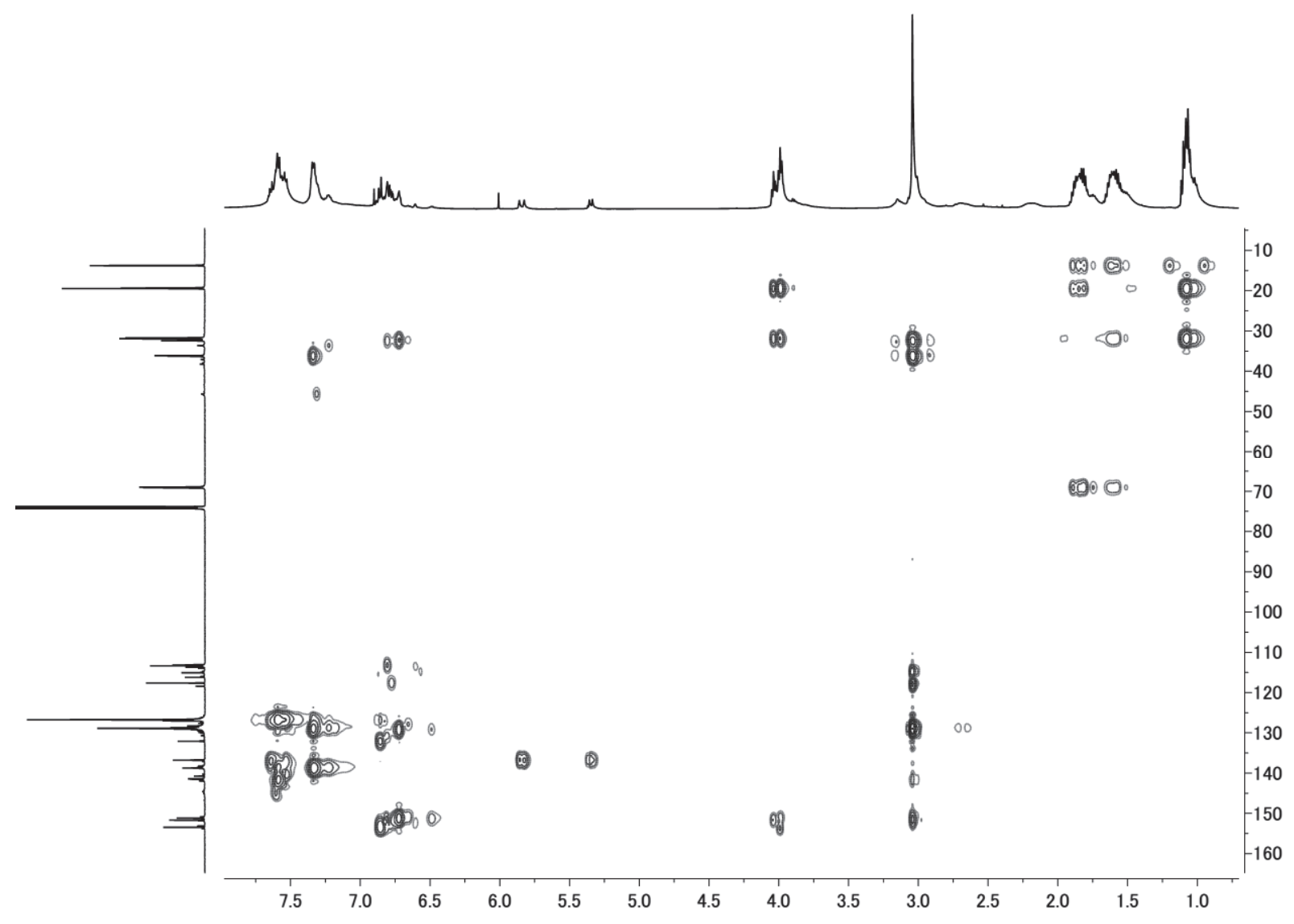

Figure S31. $\mathrm{HMBC}$ spectrum $\left(\mathrm{C}_{2} \mathrm{D}_{2} \mathrm{Cl}_{4}, 120^{\circ} \mathrm{C}\right)$ of copoly(1,4-dibutoxybenzene/4,4'-divinylbiphenyl) 

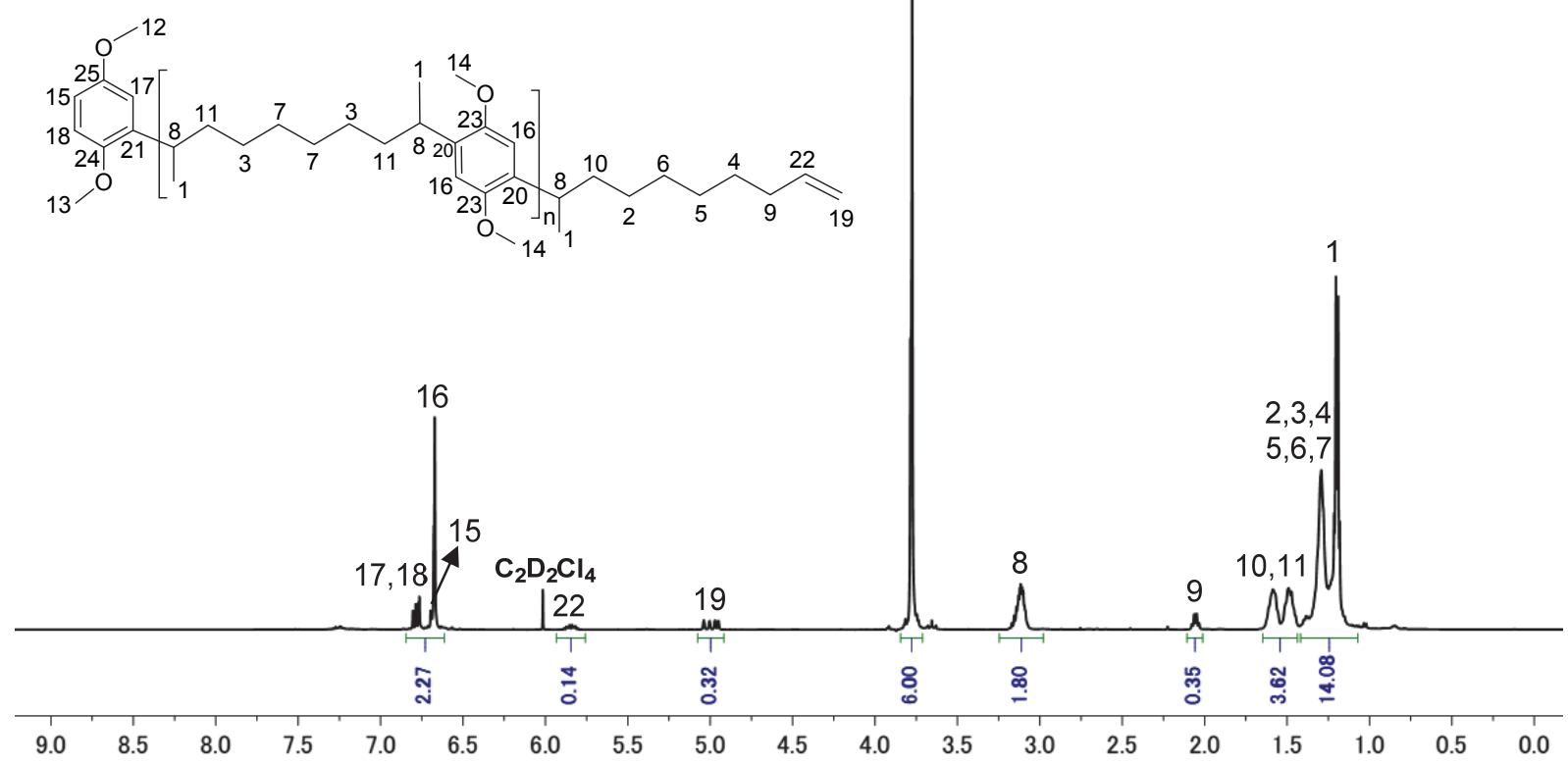

Figure S32. ${ }^{1} \mathrm{H}$ NMR spectrum $\left(500 \mathrm{MHz}, \mathrm{C}_{2} \mathrm{D}_{2} \mathrm{Cl}_{4}, 26.8{ }^{\circ} \mathrm{C}\right)$ of copoly(1,4-dimethoxybenzene/1,9decadiene)

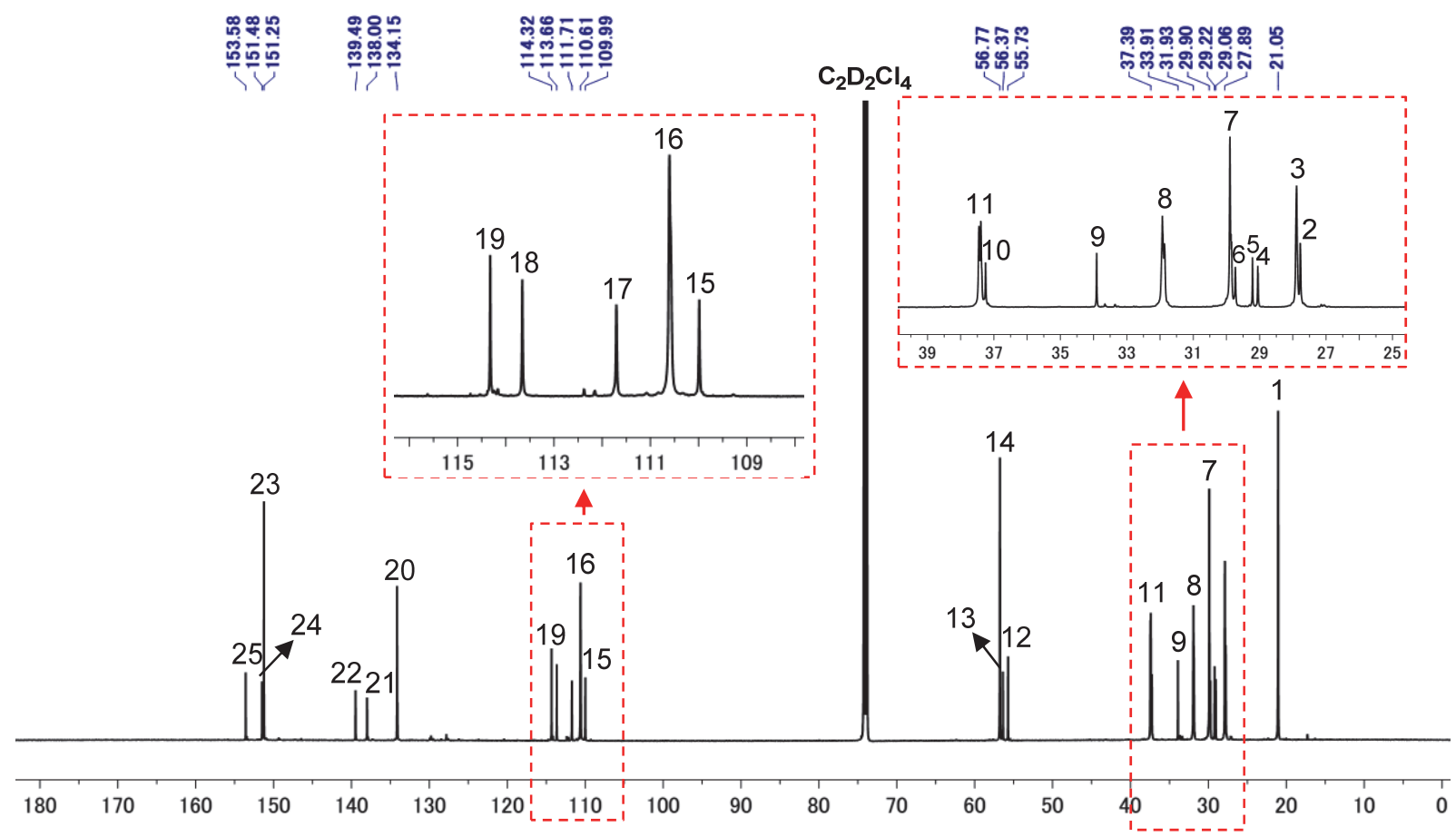

Figure S33. ${ }^{13} \mathrm{C}$ NMR spectrum $\left(125 \mathrm{MHz}, \mathrm{C}_{2} \mathrm{D}_{2} \mathrm{Cl}_{4}, 26.8{ }^{\circ} \mathrm{C}\right)$ of copoly(1,4-dimethoxybenzene/1,9decadiene) 


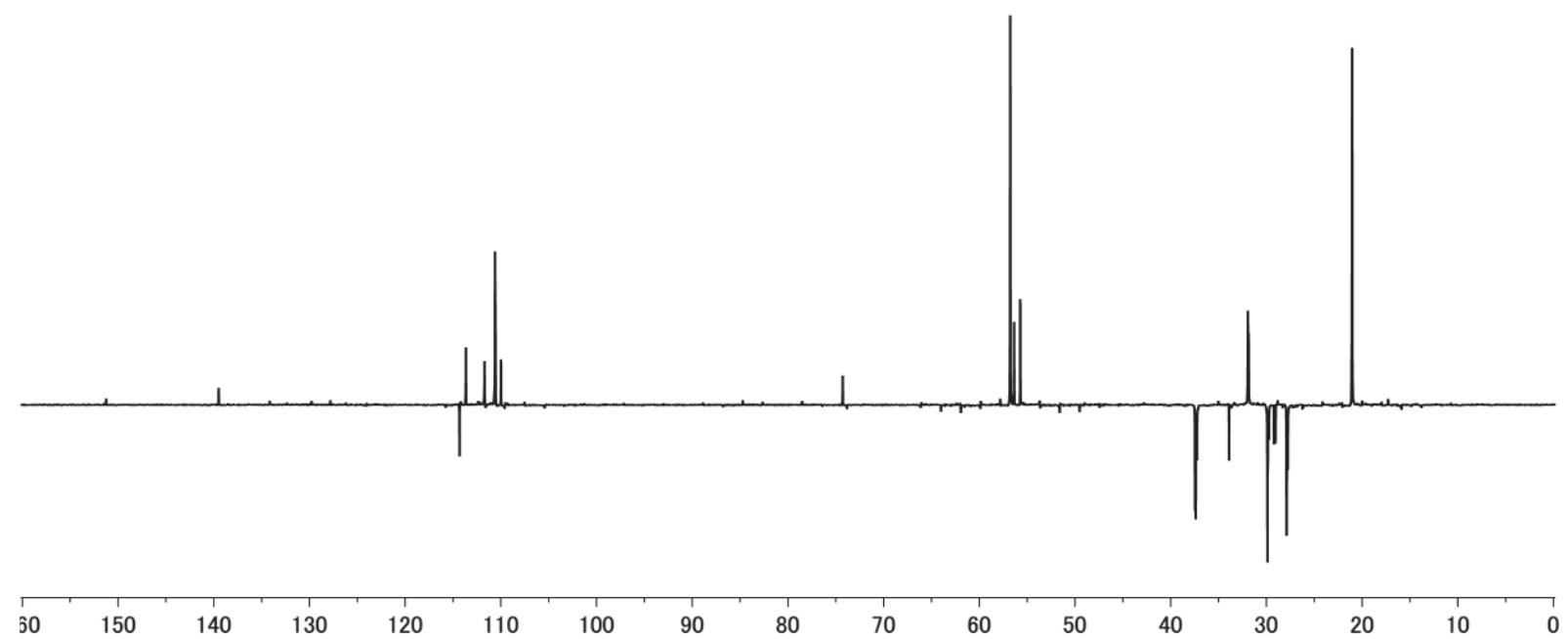

Figure S34. DEPT $135-{ }^{13} \mathrm{C}$ spectrum $\left(125 \mathrm{MHz}, \quad \mathrm{C}_{2} \mathrm{D}_{2} \mathrm{Cl}_{4}, \quad 26.8{ }^{\circ} \mathrm{C}\right)$ of $\operatorname{copoly}(1,4-$ dimethoxybenzene/1,9-decadiene)

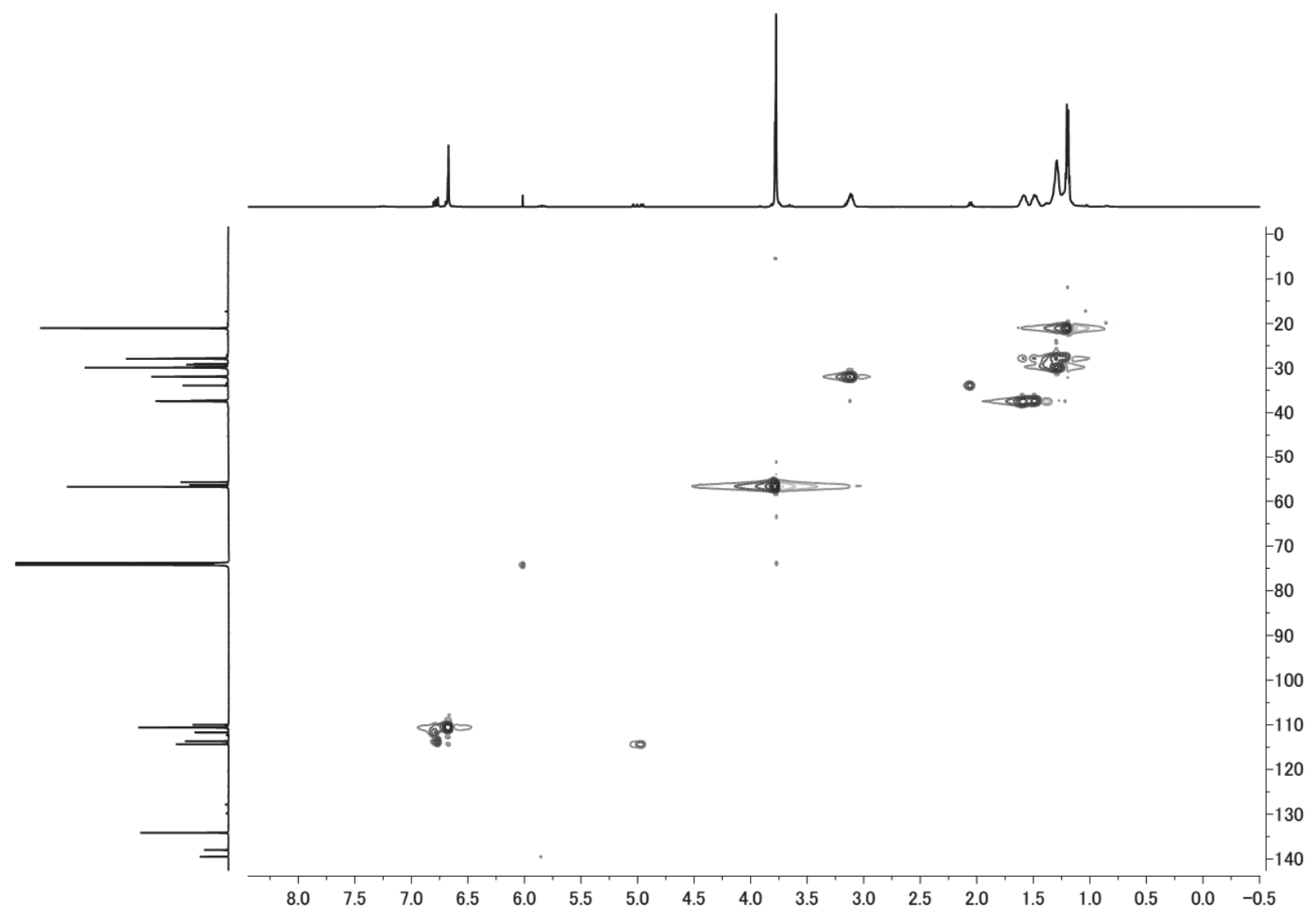

Figure S35. HSQC spectrum $\left(\mathrm{C}_{2} \mathrm{D}_{2} \mathrm{Cl}_{4}, 26.8^{\circ} \mathrm{C}\right)$ of copoly(1,4-dimethoxybenzene/1,9-decadiene) 


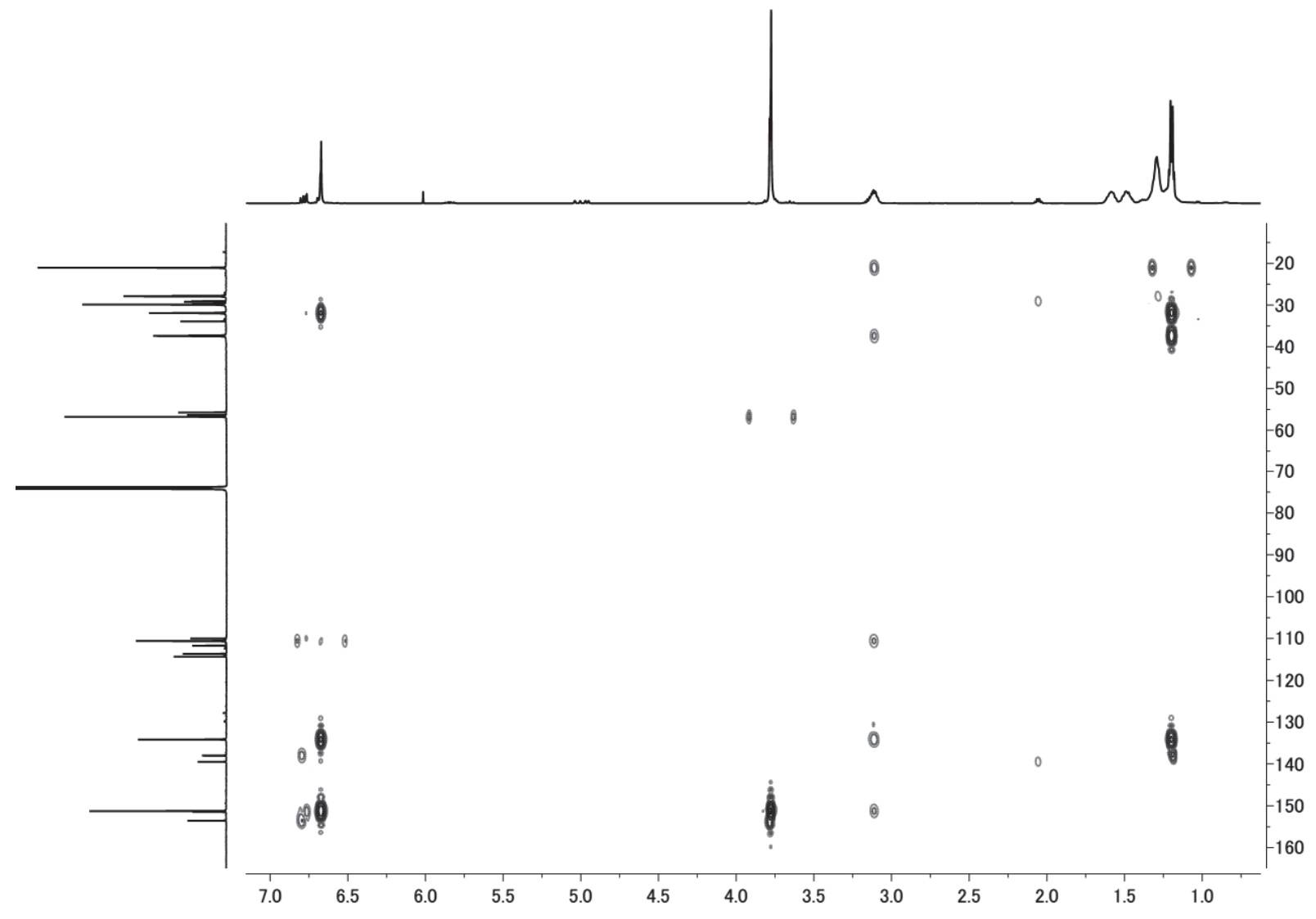

Figure S36. HMBC spectrum $\left(\mathrm{C}_{2} \mathrm{D}_{2} \mathrm{Cl}_{4}, 26.8^{\circ} \mathrm{C}\right)$ of copoly(1,4-dimethoxybenzene/1,9-decadiene) 


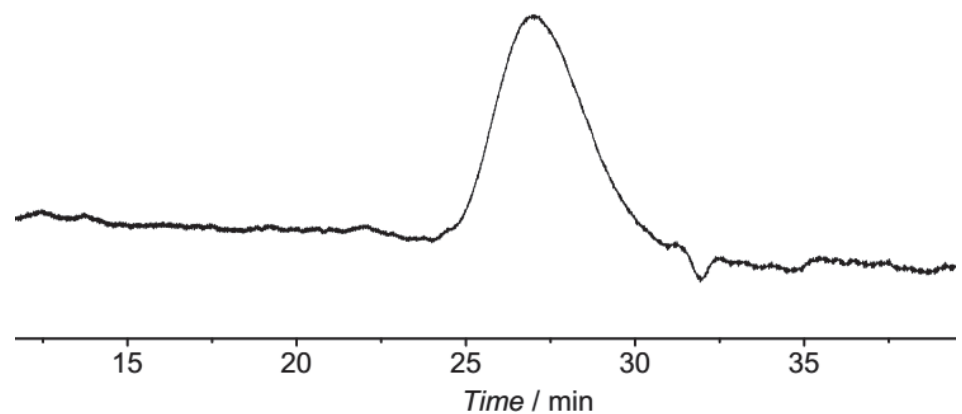

Figure S37. GPC curve of copoly(1,4-dimethoxybenzene/norbornadiene) $\left(M_{\mathrm{n}}=9800, M_{\mathrm{w}} / M_{\mathrm{n}}=2.00\right)$ in Table 1, Entry 6.

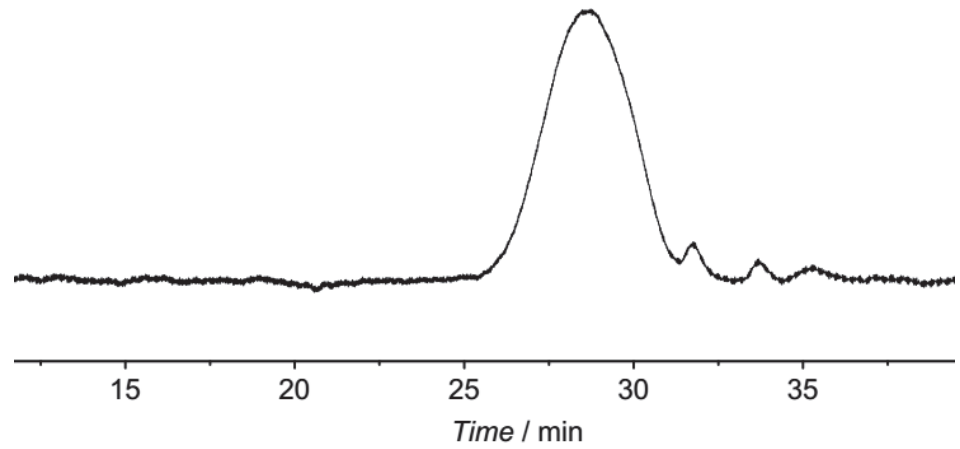

Figure S38. GPC curve of copoly(1,4-dimethoxybenzene/1,4-divinylbenzene) $\left(M_{\mathrm{n}}=3180, M_{\mathrm{w}} / M_{\mathrm{n}}=\right.$ 2.38) in Table 2, Entry 3.

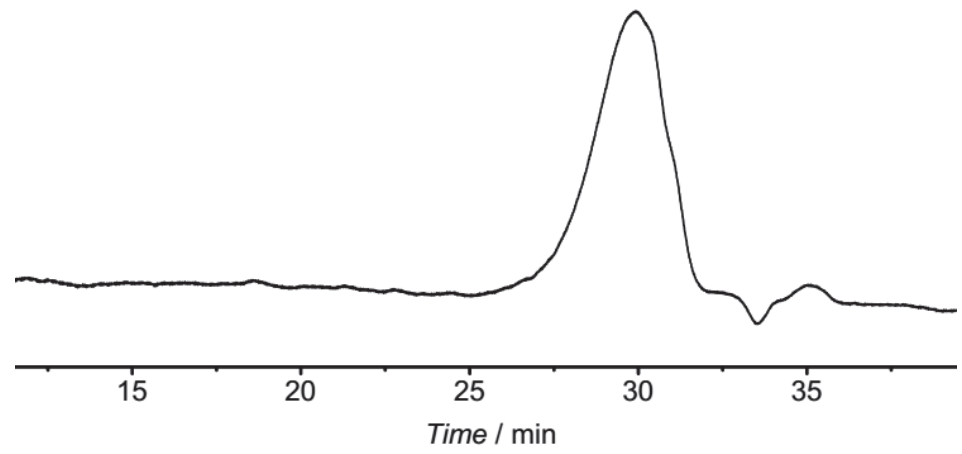

Figure S39. GPC curve of copoly(1,4-dimethoxybenzene/4,4'-divinylbiphenyl) $\left(M_{\mathrm{n}}=1900, M_{\mathrm{w}} / M_{\mathrm{n}}=\right.$ 1.94) 


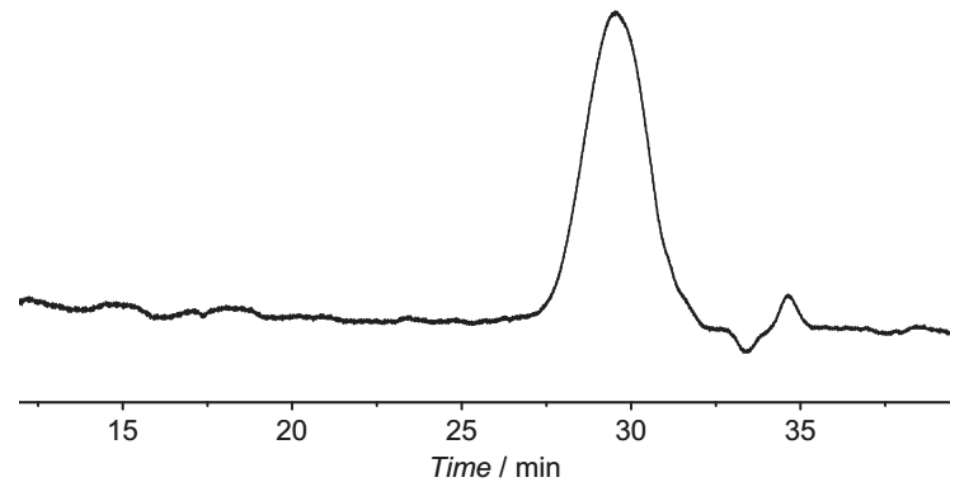

Figure S40. GPC curve of copoly $\left(4,4^{\prime}\right.$-dimethoxybiphenyl/norbornadiene) $\left(M_{\mathrm{n}}=1330, M_{\mathrm{w}} / M_{\mathrm{n}}=1.91\right)$

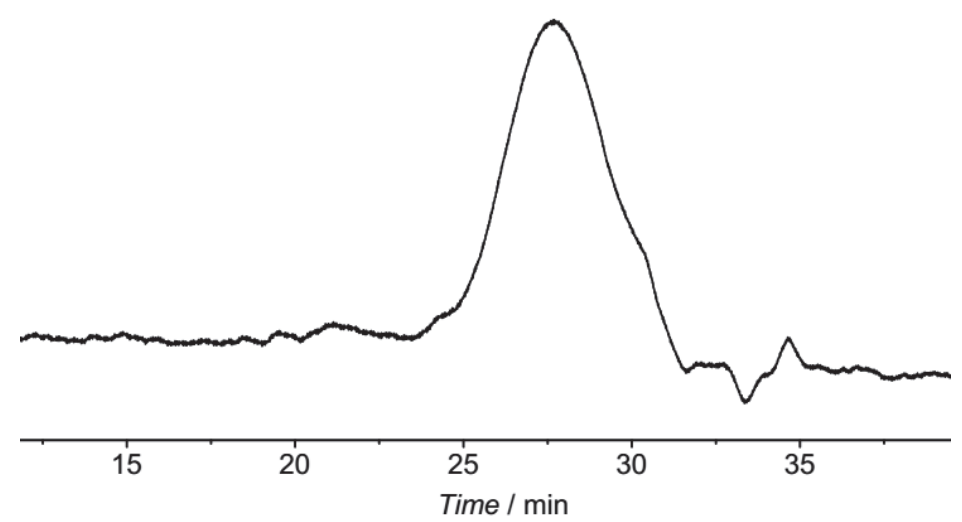

Figure S41. GPC curve of copoly(4,4'-dimethoxybiphenyl/1,4-divinylbenzene) $\left(M_{\mathrm{n}}=4550, M_{\mathrm{w}} / M_{\mathrm{n}}=\right.$ 2.52)

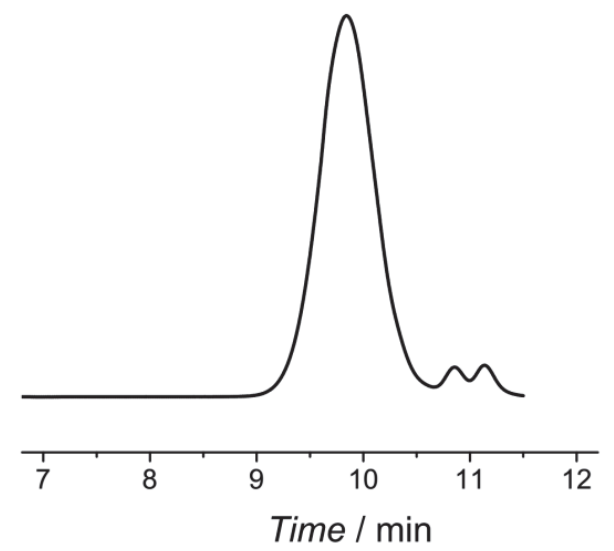

Figure S42. GPC curve of copoly(1,4-dimethoxybenzene/1,9-decadiene) $\left(M_{\mathrm{n}}=2160, M_{\mathrm{w}} / M_{\mathrm{n}}=1.23\right)$ 


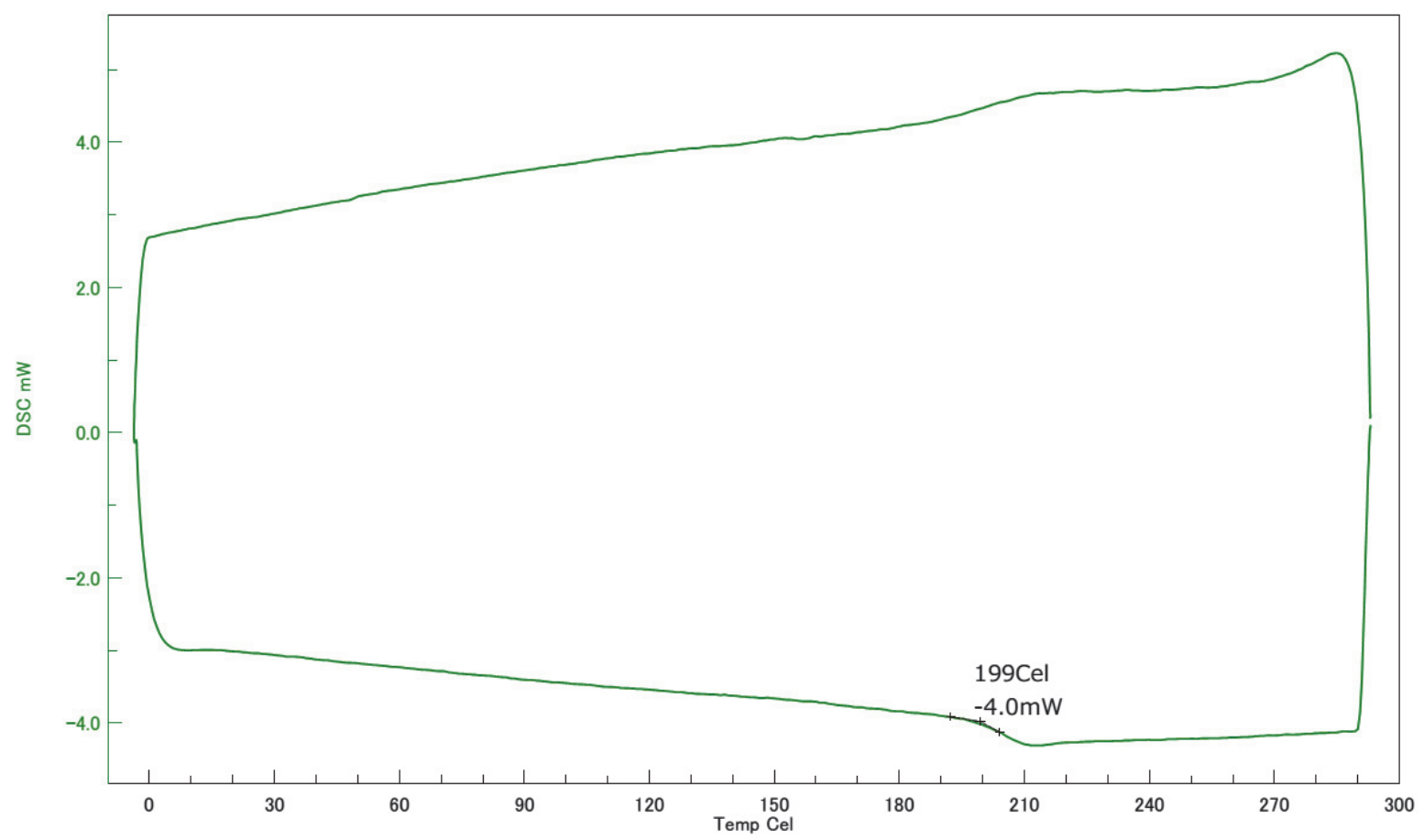

Figure S43. DSC curve of copoly(1,4-dimethoxybenzene/norbornadiene) (Table 1, Entry 6)

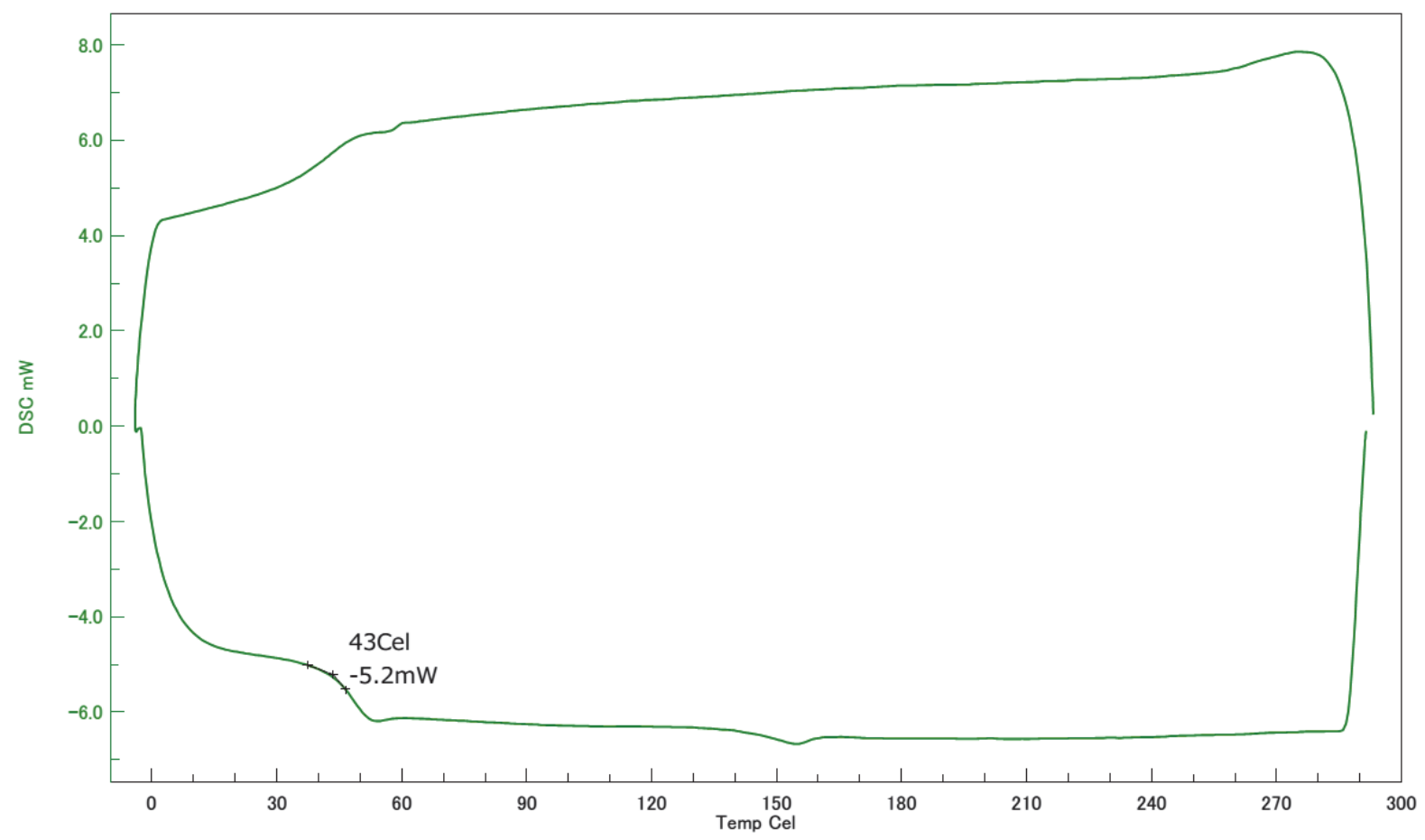

Figure S44. DSC curve of copoly(1,4-dimethoxybenzene/1,4-divinylbenzene)(Table 2, Entry 3) 


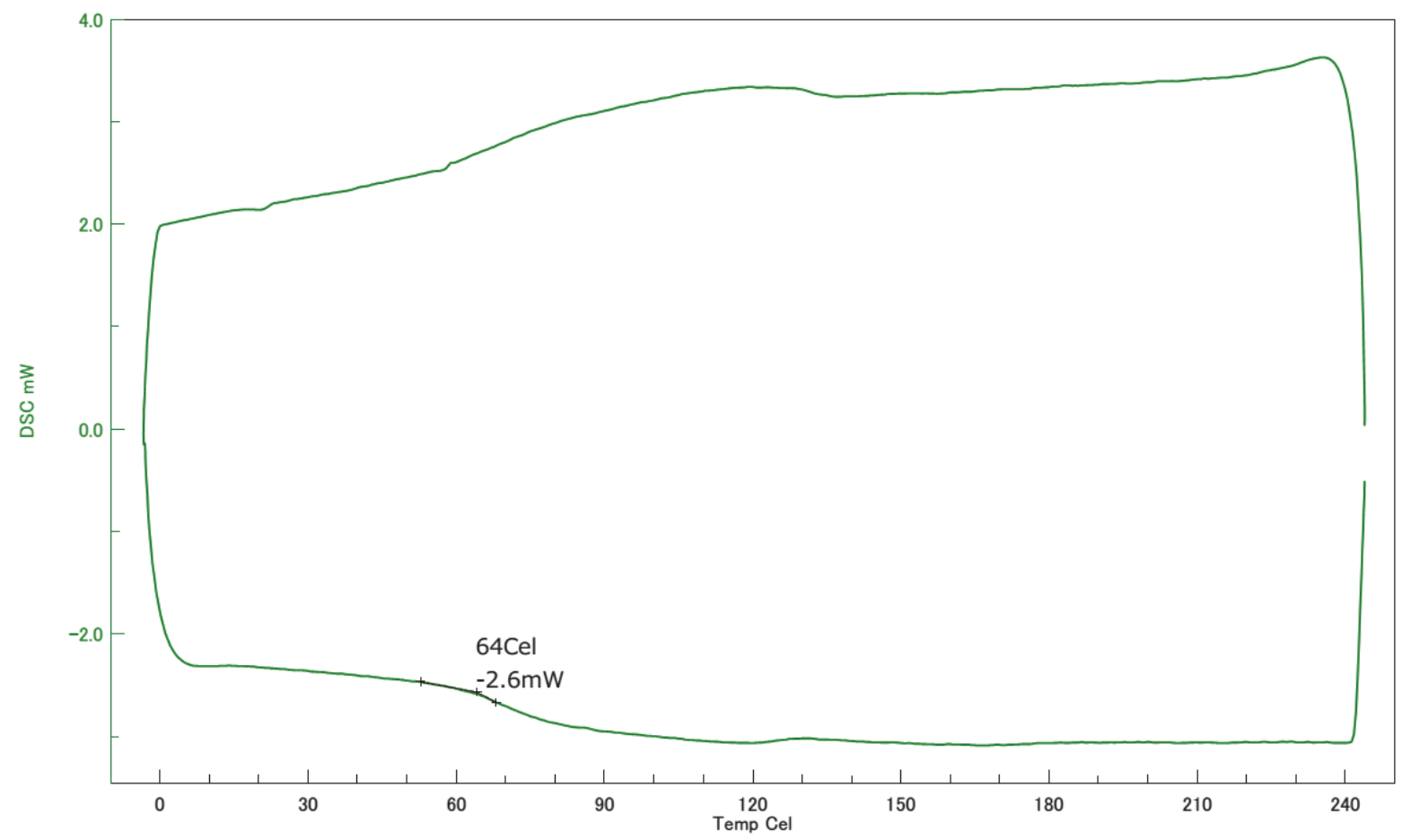

Figure S45. DSC curve of copoly(1,4-dimethoxybenzene/4,4'-divinylbiphenyl)

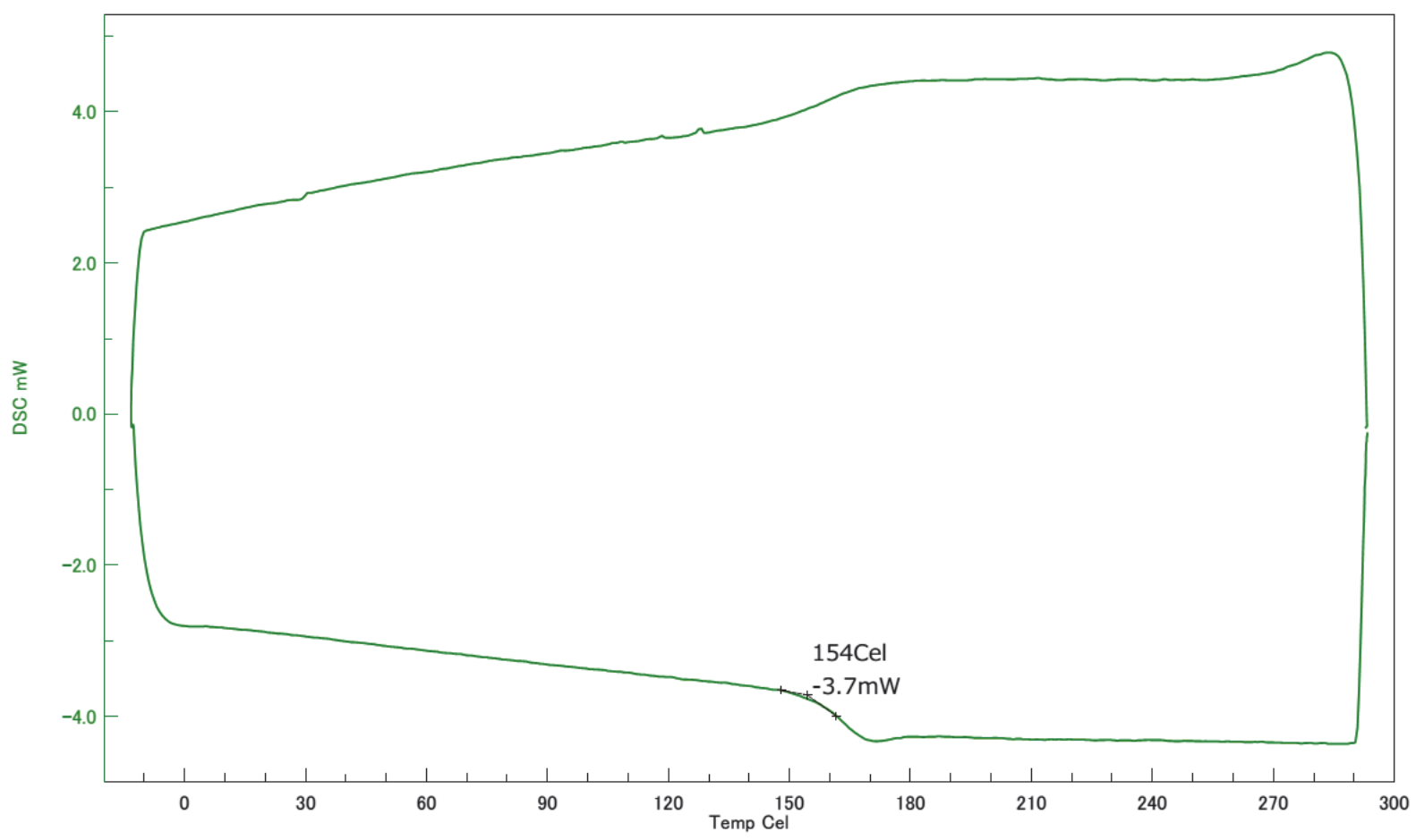

Figure S46. DSC curve of copoly(4,4'-dimethoxybiphenyl/norbornadiene) 


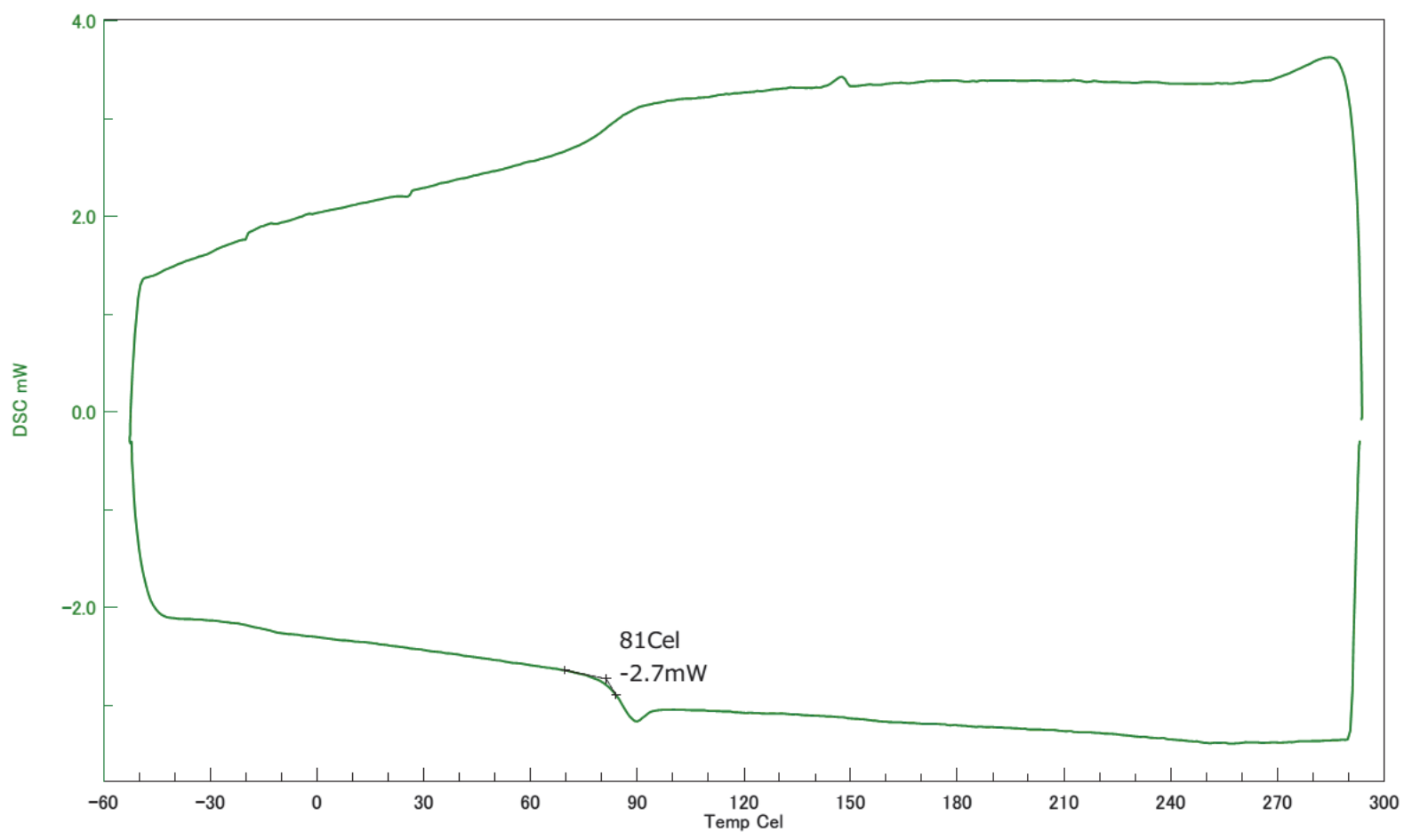

Figure S47. DSC curve of copoly(4,4'-dimethoxybipheny1/1,4-divinylbenzene)

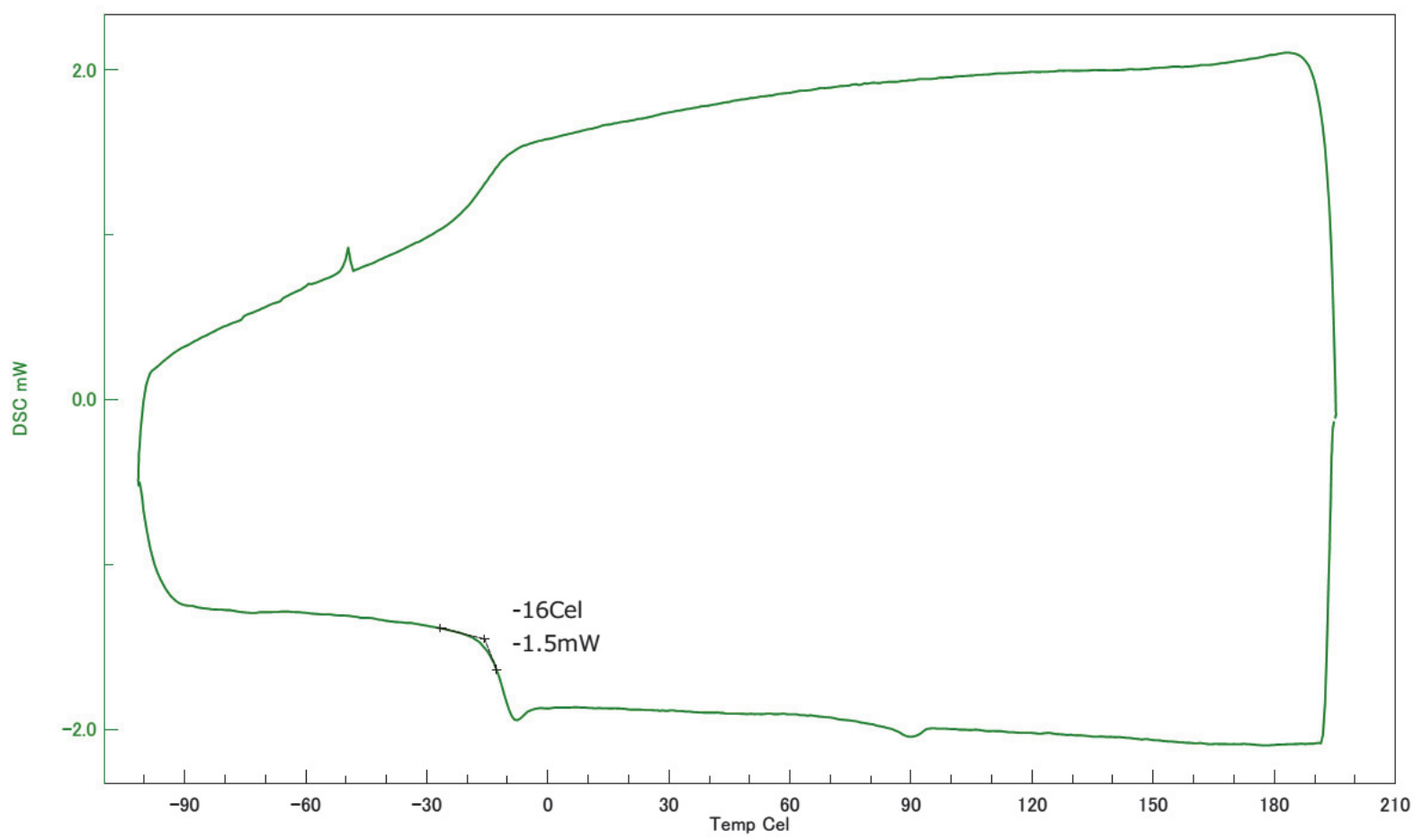

Figure S48. DSC curve of copoly(1,4-dimethoxybenzene/1,9-decadiene) 